\title{
Quality at the Source or at the End? Managing Supplier Quality Under Information Asymmetry
}

\author{
Mohammad E. Nikoofal \\ Ted Rogers School of Management, Ryerson University, Toronto, Canada, M5B 2K3, m.nikoofal@ryerson.ca \\ Mehmet Gümüş \\ Desautels Faculty of Management, McGill University, Montreal, Quebec, H3A 1G5, mehmet.gumus@mcgill.ca
}

\begin{abstract}
Despite the many benefits of outsourcing, firms are still concerned about the lack of critical information regarding both the risk levels and actions of their suppliers who are usually just a few links away. Usually, companies manage supply chain risks by deferring payments to suppliers until after the delivery has been made. Even though the deferred payment approach shunts the risk from the buyer to the supplier, recent supply chain failures suggest that it does not necessarily eliminate the risk completely. Hence, many companies offer incentives and conduct inspections of the actions taken at source rather than waiting for the end delivery. In this paper, we study the effectiveness of such incentive and inspection mechanisms undertaken by manufacturers to manage the quality of suppliers who are "privately" aware of the risk of failure. By comparing the agency costs associated with each contractual setting, we characterize the value of outputand action-based incentive mechanisms from the perspective of the manufacturer. We find that employing action-based incentives is effective for the manufacturer, specifically when working with a supplier that faces high costs of production and quality improvement. However, if the manufacturer faces high inspection costs or a low degree of information asymmetry, employing an output-based contract that results in differentiated quality improvement efforts becomes more effective. Finally, we analyze the marginal value of the combined contracting strategy and characterize when it strictly dominates over output- and effort-based contracts.
\end{abstract}

Key words: Supplier failure; Incentives; Quality improvement; Information asymmetry.

\section{Introduction}

Although outsourcing certain functions to experts brings with it economic advantages and allows the outsourcing company to focus on what it does best, it does come at the cost of an increased risk, a reduction in visibility, and a loss of control over the way in which the function is performed by the outsourcee (Knowdell 2010). A recent survey by CFO Research Services (2009) reveals that nearly $40 \%$ of companies engaging in outsourcing experience a high correlation between their global sourcing strategies and a greater risk exposure to end-product quality issues. More recent papers and surveys published as part of both academic and practitioner literature consistently show that the underlying causes behind some of these supply chain failures can be traced back to the lack 
of information regarding the suppliers' actions that have a direct impact on quality. For example, Mattel's investigation regarding the causes of its quality-related product recall in 2007 revealed that some of its contract manufacturers had intentionally avoided performing the mandated test procedures on the paint they used, which resulted in their products containing non-compliant levels of lead (Tang 2008, Babich and Tang 2012). Similarly, dozens of deaths caused by the blood thinner Heparin were traced back to a supplier in China who had used an unapproved component in order to lower production costs (Sheffi 2005).

In addition to the supplier's efforts, the quality of the end product depends on several other supply-side factors that are not easily visible to the buyer. These can include operational capability, level of experience in planning, and the execution of manufacturing. For example, one of the authors of this paper recently worked with a cable manufacturer in Europe to help minimize the impact of change-overs in the quality of custom-made cables. In the context of (electric) cable manufacturing, the quality of the end product depends on two main factors: (i) the quality of the raw materials (in this context, copper and insulation material), and (ii) the execution of the production process. The former can be assured by the cable producer by running a quality assurance (QA) test on the raw materials purchased from the suppliers. However, the latter is much more difficult to identify, because one needs to consider the entire execution of the production from the wire drawing stage all the way to packaging operation ${ }^{1}$.

Quality problems resulting from a lack of information in the supply chain have led firms using contract manufacturers to inspect their suppliers' end products and their production-related decisions and link their incentives to the outcomes of these inspections. For example, after Volvo's recall of certain products due to malfunctioning fuel pumps (Harley Decebmer 2009), it established a Supplier Evaluation Model under which all existing suppliers are tested to verify whether their end products meet the pre-specified quality and reliability standards. Suppliers are also awarded with additional business if their combined scores pass a certain level (Volvo 2010). Also, after its 2007 product recall, Mattel started to release payments to its vendors if and only if they had purchased their components from a list of certified suppliers, who had been pre-audited to ensure compliance with lead level standards (Tang 2008). These examples are not only specific to companies that have had to undertake a product recall. Verizon Wireless, a major carrier serving more than 62 million customers in the US, performs extensive and costly testing activities on new phones received from major manufacturers such as Motorola, Samsung, and LG Electronics.

\footnotetext{
${ }^{1}$ There are many subtleties in the execution system that affect the end quality of the cables. For example, the more frequently the cable type is changed (e.g., cables with a larger nominal diameter being replaced with cables of a smaller nominal diameter), the lower will be the consistency of the end product and, in turn, the lower will be the quality of the cables.
} 
There are two types of inspections: (i) to test whether the quality of the final output conforms to the standards pre-specified by the manufacturer, and (ii) to test the suppliers' actions that affect the quality of the final product (Dyer et al. 1998). Because of the very existence of supply-side factors that cannot be readily observed by the manufacturer, there is no one-to-one correspondence between the supplier's efforts and the quality of the end product. Hence, each inspection mechanism provides the manufacturer with a different type of information. While the first type of inspection provides the manufacturer with information regarding the quality of the end product, the second type enhances the visibility of the efforts made by the supplier. Furthermore, in either case, the manufacturer would incur a cost to receive (potentially inaccurate) information. Even though most of the recent papers in the literature on supply chains study inspection mechanisms to test the quality of the end product (see Babich and Tang (2012), Rui and Lai (2015) and references therein), to the best of our knowledge, no study has evaluated and compared the two inspection mechanisms mentioned above and their impact on supply chain performance under information asymmetry.

To address this gap, in this paper, we compare two incentive and inspection mechanisms: $(i)$ in the first mechanism, the manufacturer offers the supplier a menu of contracts that is contingent on the end product's quality (hereafter referred to as "Quality-at-the-End (QE) contract"), and; (ii) in the second mechanism, the payment is released to the supplier based on the actual quality-effort decision made at source (hereafter referred to as "Quality-at-the-Source (QS) contract"). In both cases, the manufacturer also decides whether or not to inspect the supplier before releasing the contingent payment. Building on these two mechanisms, our aim is to answer the following research questions:

Research Question 1: What is the impact of the optimal QE and QS contracts on the incentives of the manufacturer and the supplier as well as the reliability of the entire supply chain?

Research Question 2: Which one of the above incentive and inspection mechanisms should be employed by a manufacturer in dealing with a risky supplier?

Research Question 3: How does asymmetric information affect the value of each contracting strategy from the perspective of the individual supply chain parties as well as the supply chain as a whole?

In order to answer the above research questions, in this paper, we analyze a decentralized supply chain model in which a manufacturer procures from a supplier whose production process is subject to external quality risk, the extent of which is private information for the supplier. Furthermore, in order to reduce quality risks, the supplier can make expensive quality improvement effort that is also unobserved by the manufacturer. Building on this modeling framework, we consider two contracting strategies between manufacturers and suppliers. As per the QE contract, a manufacturer's payments to its supplier are contingent on the end-quality of the delivered product. As per the QS contract, the 
manufacturer ties payments to the level of effort made by the supplier. Depending on the contracting stage, the supplier decides whether to make a quality improvement effort and, subsequently, the manufacturer decides whether or not to conduct an inspection of the supplier. As per the QS contract, an inspection involves auditing the quality improvement effort made by the supplier, while the QE contract involves testing the quality of the end product itself. The outcomes of both inspection and audit tests are inaccurate. Also, if the manufacturer decides not to conduct a test, it must make the contingent payment to the supplier. Based on this mechanism, we define optimal QE and QS contracts and equilibrium decisions as well as determine the optimal contracting strategy from a manufacturer's perspective, by comparing the equilibrium payoffs under the optimal QE contract to those under the optimal QS contract.

Our comparative analyses have helped establish that as a supplier's production and quality improvement costs increase, the action-based optimal QS contract becomes a more effective contracting strategy for a manufacturer. This is because the QS contract subsidizes the cost of the supplier completely, given that it is the supplier that makes the quality improvement effort. As the costs of inspection incurred by the manufacturer increase and/or the degree of information asymmetry between the manufacturer and the supplier decreases, the output-based optimal QE contract becomes more effective for the manufacturer. Under the optimal QE contract, the manufacturer avoids having to make a contingent payment if the quality of the outcome does not match the required specifications. Hence, compared to the optimal QS contract, the optimal QE contract benefits the manufacturer because of its lower monitoring costs. Finally, we show that a combined contract proves to be more effective than both the optimal QE and QS contracts when the degree of accuracy of both inspection and audit tests is relatively low. Combining information from inspections and audits helps the manufacturer enhance the degree of accuracy, which makes it easier for her to induce quality improvement effort on the supplier.

\section{Literature Review}

Our paper is related to two streams of research in the field of operations management. The first focuses on modeling quality improvement decisions in supply chain firms. The second stream relates to contract design under supply disruption. Below, we review each of these streams and show how they are related to our work.

Papers focusing on the first stream of quality improvement vary in terms of whether the quality improvement effort is subject to the single-moral hazard problem (Babich and Tang 2012, Baiman et al. 2003), the double-moral hazard problem (Reyniers and Tapiero 1995, Baiman et al. 2000, Balachandran and Radhakrishnan 2005), or a mixed model of adverse selection followed by moral hazard problem (Kaya and Özer 2009, Chao et al. 2009). This paper is more in line with mixed 
models because, in our model, the supplier's extent of reliability as well as the choice of improvement efforts may not be observable by the manufacturer. The paper by Kaya and Özer (2009) explores how the manufacturer's pricing decision may affect quality risk where the quality is determined solely by the supplier and directly affects market demand. Our paper differs from Kaya and Özer (2009) in three main ways: First, in our model, the supplier is paid based on either the quality of the product (the QE contract) or the level of effort made to maintain quality (the QS contract). Kaya and Özer (2009) however assumes that quality is not contractible; hence, the manufacturer cannot link the compensation of the supplier to the quality of the product. Second, in our model, the supplier's private information is defined on his reliability, while, in the paper by Kaya and Özer (2009), it is defined on the cost of quality. This leads to a fundamental difference in the analysis. Specifically, the quality level assumed by the supplier in Kaya and Özer (2009) does not change the cost of ensuring quality and, thus, has no effect on the degree of information asymmetry between different supplier types. Conversely, in our model, the extent of improvement measures taken by the supplier affects its reliability and, hence, affects the degree of information asymmetry between the manufacturer and the supplier. Finally, this paper focuses on evaluating two contractual strategies from the manufacturer's perspective, aimed at managing the supplier's quality improvement effort, while Kaya and Özer (2009) focuses on the impact of the manufacturer's pricing decisions on the supplier's quality decisions.

The modeling framework employed in our paper is similar to Babich and Tang (2012) and Rui and Lai (2015) in relation to the use of inspections whereby the manufacturer endogenizes the testing and quality improvement efforts made by the supplier. However, our paper differs from these studies in four ways: First, in Babich and Tang (2012) and Rui and Lai (2015), the supplier's actions deterministically affect the quality of the end product, while in our model, the quality of the end product is stochastically influenced by the supplier's actions as well as the supplier type. Therefore, in Babich and Tang (2012) and Rui and Lai (2015), inspecting the end-quality is equivalent to auditing the supplier's actions, while this is not the case in our model. Second, Babich and Tang (2012) and Rui and Lai (2015) compare two incentive mechanisms - delayed payment and inspection - that differ from each other in terms of when the inspection is conducted. In the first mechanism, the inspection is conducted (by the customers) after the end product has been delivered, whereas in the second mechanism, the inspection is conducted (by the buyer) before the end product has been delivered to the customer. Conversely, we compare two incentive mechanisms - quality-at-the-end (QE) and quality-at-the-source (QS) - that differ from each other in terms of what is being inspected. Since the effort deterministically affects the quality of the end product, the question of "what" (is being inspected) does not play any role in Babich and Tang (2012) and Rui and Lai (2015). Third, our model is built on adverse selection followed by a moral hazard, whereas Babich and Tang 
(2012) and Rui and Lai (2015) consider only the moral hazard. Therefore, we explicitly consider and compare the impact of information asymmetry on the different contracting strategies. Please refer to the characterization of information rent and channel loss in $\S 4$ and $\S 5$ as per the optimal QE and QS contracts, respectively, for details. Finally, while we consider the degree of accuracy on two different mechanisms - inspections and audits - the degree of accuracy is modelled only for the inspection mechanism in Babich and Tang (2012) and Rui and Lai (2015). Combining the information from both inspections and audits, the manufacturer can improve the overall accuracy of the information. This in turn enables the manufacturer to induce the supplier to make quality improvement efforts, even when the information derived from the inspections and audits is not very accurate.

Papers on contract design under supply disruption study hidden information problems in the supply chain context (see Cachon 2003 for an excellent review of this literature). The papers in this stream can also be categorized in terms of whether the hidden information is defined in terms of the supplier's cost (e.g., Corbett et al. 2004, Cachon and Zhang 2006, Özer and Raz 2011, Kim and Netessine 2013) or reliability (e.g.Yang et al. 2009, Tomlin 2009, Chaturvedi and Martinez-de Albeniz 2011, Gümüş et al. 2012, Yang et al. 2012). Our paper contributes to the study of supplier reliability by developing a supply chain reliability model wherein the manufacturer suffers on account of the unobservability of both the supplier's reliability (hidden information) and improvement effort (hidden action). A paper in this stream that is most similar to ours is Yang et al. (2009). This paper considers the value of backup production in a supply disruption setting where the reliability of the supplier is known only to the supplier. Our paper differs from Yang et al. (2009) in two main ways: First, we consider the supplier's hidden actions in addition to his private information. Second, the backup production option explored in Yang et al. (2009) is a contingency tactic, which can be employed by the supplier in the case of disruption; however, quality improvement is a mitigation tactic that reduces exposure to disruption.

Our paper is also related to the design of sales-force compensation schemes that have been extensively analyzed in economics, marketing, organizational behaviour, and CEO compensation schemes in finance and accounting. Similar to the QE and the QS contracts considered in this paper, two incentive mechanisms have been proposed in the literature on sales forces: (i) an output-based compensation scheme that makes the salesperson's income a function of sales (e.g., Basu et al. 1985, Raju and Srinivasan 1996) and (ii) a behavior-based compensation scheme that involves monitoring the salesperson's efforts and linking his or her income to the level of effort put into the selling task (e.g., Anderson and Oliver 1987, Joseph and Thevaranjan 1998). Similarly, "output-based" and "behaviour-based" incentives, which correspond to the QE and QS contracts in our model, respectively, have been proposed to control the managerial efforts made by CEOs (Murphy 1999, Makri 
et al. 2006). Building on theoretical and empirical models, these papers establish different market conditions under which each control strategy is more valuable for the stake-holders. In the context of both sales force and CEO compensation, the appropriate control strategy depends on several domain-specific factors that are not considered in our model, such as sales volatility, expectancy (Basu et al. 1985), and the degree of substitution and complementarity between products (Coughlan and Sen 1989) in sales force compensation, as well as the degree of technological intensity (Makri et al. 2006) in CEO compensation in technology-intensive firms. That said, the models proposed in these papers have considered some factors that have also been analyzed in our paper. Among them are the costs associated with outcome and behaviour measurements. In the literature on sales force compensation in particular, it has been shown that as the cost of measurement increases, the output-based control strategy, which corresponds to our QE contract, becomes more beneficial for the firm (Anderson and Oliver 1987). This is also in alignment with our findings, in the sense that the manufacturer would prefer the optimal QE contract over the optimal QS contract when the cost of inspection is high.

Lastly, our model is built on the joint adverse selection and moral hazard framework on which there is extensive literature in the field of economics, which has been developed following the seminal paper by Laffont and Tirole (1986). We extend the mixed modelling framework by incorporating inspection/audit sub-games and apply it to supply risk management. For the numerous applications of the mixed modelling framework, we refer to Laffont and Martimort (2002), Anthon et al. (2010) and references therein.

\section{Model Framework}

In order to address the research questions raised in $§ 1$, we develop a two-stage supply chain model between a manufacturer (hereafter referred to as "she") and a risky supplier (hereafter referred to as "he"). In order to satisfy the downstream demand for the end product, the manufacturer requires a component from an upstream party (i.e., the supplier). To focus on the supply-side risks, we assume that the demand for the end product is known and, without loss of generality, has been normalized to 1 . The component is produced by the supplier at $c$ per unit. The supplier's production is exposed to quality risks, denoted by a random variable $\tilde{q}$. Namely, with some probability, the component produced by the supplier fails to conform to the specifications of the manufacturer and hence results in the failure of the end product. Note that in the literature on reliability, such a product failure is defined as the "weakest link property" and is one of the most common causes of product failure (Chao et al. 2009, Baiman et al. 2004). More specifically, a survey by the global management consulting firm A.T. Kearney Inc. showed that $21 \%$ of the external quality failures in North America's auto industry in 2002 were related to the supplier's manufacturing process, while $15 \%$ were due to the manufacturer's process. 
We assume that the extent of the supplier's failure risk depends on two factors: The first is exogenous to the supplier, which ultimately relates to the degree of the supplier's reliability. We define this by $\theta$ and, for the sake of analytical tractability, assume that $\theta$ can have two values $-h$ and $l$, representing $h$ - and $l$-type suppliers, respectively. Keeping all else equal, the $l$-type supplier faces more (exogenous) failure risks than the $h$-type in the sense of first-order stochastic dominance (to be formally defined in Assumption 1). Second, we consider endogenous factors that can be actively managed by the supplier in order to reduce his exposure to failure risks. For example, a component manufacturer may make various pre-emptive quality control efforts in order to detect and fix process-related problems before the start of a (costly) operation. In the literature on quality control, such actions are generally implemented by conducting a failure mode and effect analysis (FMEA) (Stamatis 2003). We denote all such improvement efforts conducted by the $\theta$-type supplier before the start of production by $e_{\theta} \in\{0,1\}$. For analytical tractability, we assume that the cost of making quality improvement efforts is $C_{q}$ for both $\theta \in\{l, h\}$.

Next, we model the effect of the exogenous $(\theta)$ and endogenous $\left(e_{\theta}\right)$ factors on the probabilities of survival and failure events in the end product via $p_{\theta}\left(e_{\theta}\right)$ and $1-p_{\theta}\left(e_{\theta}\right)$, respectively. Let $\tilde{q}_{\theta}$ be the realized outcome for the component produced by the $\theta$-type supplier. Then, it can be characterized by a Bernoulli random variable with the following probability mass function:

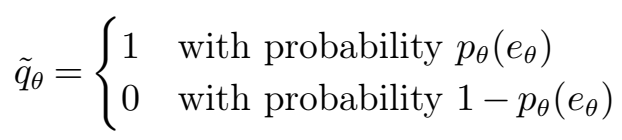

where $\tilde{q}_{\theta}=0$ and $\tilde{q}_{\theta}=1$ represent non-conformance and conformance events, respectively. Note that a binary outcome (pass or fail) is quite common in quality control. Specifically, a variety of chartbased quality control techniques have been developed to have "in-control" and "out-of-control" outcomes, based on appropriately defined upper- and lower-control limits. We refer to Baiman et al. (2004) and the references therein for binary models of threshold-based quality control policies between manufacturers and suppliers.

As mentioned above, quality improvement efforts help the supplier to increase the likelihood of conformance (i.e., $\tilde{q}_{\theta}=1$ ). For the sake of analytical tractability, we set the change in the likelihood of the conformance outcome due to quality improvement efforts that are the same for both supplier types $-p_{h}(1)-p_{h}(0)=p_{l}(1)-p_{l}(0)>0$. The conditions mentioned below characterize the impact of the supplier's reliability type and efforts on the likelihood of conformance:

\section{Assumption 1.}

(A) For the same level of effort, i.e., $e_{h}=e_{l}=e \in\{0,1\}$, the probability of the conformance of the component produced by the more reliable supplier (i.e., $h$-type) is always larger than that for the less reliable one (i.e., l-type). 
Nikoofal and Gümüş: Quality at the Source or at the End?

(B) The probability of the conformance of the components produced by both $l$ - and $h$-type suppliers increases as per their quality improvement efforts $e_{\theta}$.

The first condition essentially implies that $p_{h}(1) \geq p_{l}(1)$ and $p_{h}(0) \geq p_{l}(0)$, and the second implies that $p_{\theta}(1) \geq p_{\theta}(0)$ for both $\theta=\{l, h\}$. The above conditions are standard in the literature on information asymmetry, wherein they are commonly referred to as Spence-Mirrlees Conditions (Laffont and Martimort 2002).

Next, we discuss the distribution of information among the supply chain parties. Due to the asymmetry of information between the manufacturer and the supplier, the true type of the supplier's reliability as well as the quality improvement effort made by him are only known to the supplier himself. This essentially means that the manufacturer has only a-priori beliefs regarding the true type of the supplier. We denote the manufacturer's a-priori beliefs on true type $\theta$ by $\nu_{\theta} \in[0,1]$, where $\nu_{h}=\nu$ and $\nu_{l}=1-\nu$ represent the likelihood of $h$ - and $l$-type suppliers, respectively. Finally, we assume that all these parameters are common knowledge among all the players.

Using the above modeling framework, we evaluate two different inspection policies and contractual offerings provided by the manufacturer to the supplier in order to control his quality improvement when there are quality risks under information asymmetry. In the first setting, the manufacturer performs an expensive quality inspection test on the supplier's production outcomes and offers a contract contingent on the conformance of the component produced by the supplier. Hereafter, we call this the QE (Quality-at-the-End) contract. In the second setting, the manufacturer performs an expensive audit to verify the supplier's quality improvement efforts and ties the contract terms to whether or not the supplier makes a specific quality improvement effort at source. Hereafter, we call this the QS (Quality-at-the-Source) contract. The outcomes of both inspection and audit tests are inaccurate. We will provide the modelling details for the accuracy of inspection and audit tests in $\S 4$ and $\S 5$, respectively.

The QE contract has two terms. The first is an upfront transfer payment denoted by $\omega_{\theta}$, which is payable by the manufacturer to the $\theta$-type supplier upon the supplier's participation. The second term is contingent payment denoted by $Y_{\theta}$ and is payable to the supplier at the end of production, depending on the end-quality of the product and the inspection test report. Specifically, if the manufacturer performs the test, the supplier will get paid only if the production outcome passes the quality test. However, if the manufacturer does not perform the test, the contingent payment is made to the supplier without any further conditions.

The QS contract also has an upfront transfer payment term, denoted by $\omega_{\theta}$, which is paid to the supplier upon his participation. However, under the QS contract, the manufacturer can potentially observe the $\theta$-type supplier's quality improvement efforts $e_{\theta}$; hence, the supplier's efforts are included in the contract terms as $E_{\theta} \in\{0,1\}$. Therefore, the contingent payment, denoted by $Y_{\theta}$, is transferred 
to the supplier based on the manufacturer's audit and the supplier's decisions with regard to quality improvement efforts. More specifically, if the manufacturer audits the supplier's efforts, she would make the contingent payment to the supplier if and only if the quality improvement efforts made by the supplier correspond to those mentioned in the contract, i.e., if $e_{\theta}=E_{\theta}$. If the manufacturer does not conduct any audit, the supplier is paid without any conditions.

Since under both contracts the supplier's true type of reliability is unknown to the manufacturer, she has to design a menu of contracts from which each supplier type self-selects one designed for him. Invoking the extended revelation principle for mixed adverse selection and moral hazard problems (see Myerson (1982)), without loss of generality, we can restrict our attention to direct-revelation mechanisms in which the suppliers truthfully reveal their types and perform actions that are induced by the manufacturer. Thus, it is sufficient for the manufacturer to offer two contracts, one for each type, i.e., $\left(\omega_{h}, Y_{h}\right)$ and $\left(\omega_{l}, Y_{l}\right)$ under the QE contract, and $\left(\omega_{h}, Y_{h}, E_{h}\right)$ and $\left(\omega_{l}, Y_{l}, E_{l}\right)$ under the QS contract. Below, we provide the timing of events and actions (see Figure 1).

- At time zero, the supplier observes the true type of reliability, i.e., $\theta \in\{l, h\}$.

- The manufacturer chooses her contracting strategy, selecting between the QE and QS contracts.

- The manufacturer offers a menu of contracts, i.e., $\left(\omega_{h}, Y_{h}\right)$ and $\left(\omega_{l}, Y_{l}\right)$ under the QE contract, and $\left(\omega_{h}, Y_{h}, E_{h}\right)$ and $\left(\omega_{l}, Y_{l}, E_{l}\right)$ under the QS contract.

- The $\theta$-type supplier then self-selects a contract designed for him from the menu and receives an upfront payment $\omega_{\theta}$.

- The $\theta$-type supplier decides whether or not to make quality improvement efforts, i.e., $e_{\theta} \in\{0,1\}$.

- If the QS contract is offered, the manufacturer decides whether or not to audit the supplier's quality improvement efforts before production begins. If she performs an audit, she makes the contingent payment $Y_{\theta}$ if and only if $e_{\theta}=E_{\theta}$. If she decides not to conduct an audit, she makes the contingent payment $Y_{\theta}$ without any conditions.

- The supplier completes the production and the end-quality of the component $\tilde{q}_{\theta} \in\{0,1\}$ is realized.

- If the QE contract is offered, the manufacturer decides whether or not to inspect the production outcome in order to verify if the component produced by the supplier conforms to the specifications. If she decides to conduct an inspection, she makes the contingent payment, i.e., $Y_{\theta}$ if and only if $\tilde{q}_{\theta}=1$. If she decides not to conduct an inspection, she makes the contingent payment to the supplier regardless of the end product's quality.

Before considering information asymmetry, we first analyze the problem in the context of complete information. We specifically consider a case in which the manufacturer and the supplier work together as an "integrated firm" and make quality improvement decisions in a centralized fashion. Let "fb" indicate the first-best level of efforts made under such conditions. The integrated firm's 


\section{Figure 1 Sequence of events}

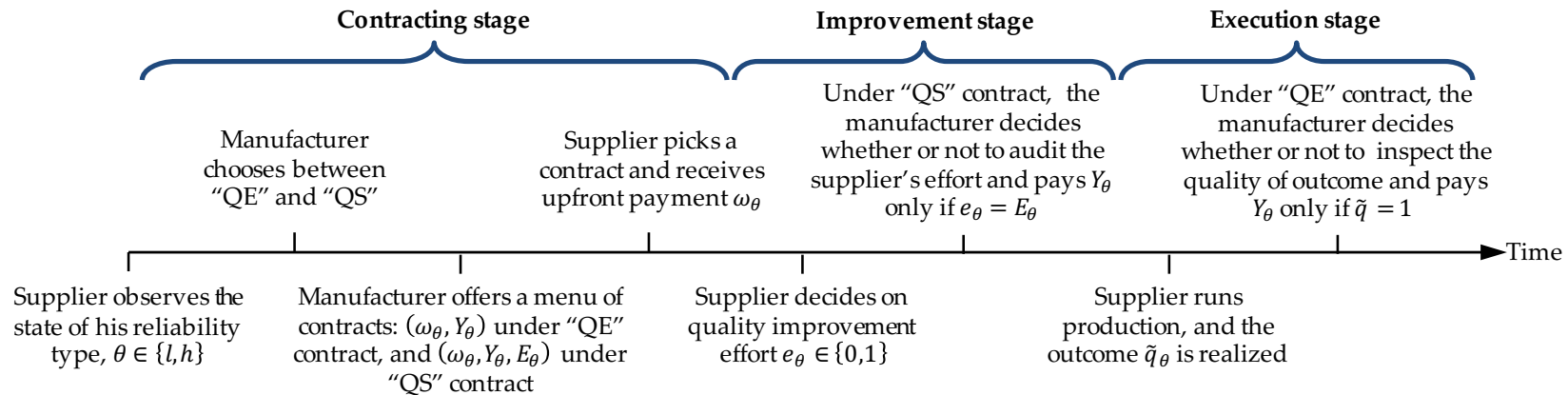

optimization problem is then to find the optimal level of improvement efforts $e_{\theta}^{\mathrm{fb}} \in\{0,1\}$ that would maximize its profit. If the integrated firm invests in quality improvement efforts (i.e., $e_{\theta}^{\mathrm{fb}}=1$ ), its expected profit is $p_{\theta}(1) r-c-C_{q}$. If it does not make such efforts (i.e., $e_{\theta}^{\mathrm{fb}}=0$ ), its expected profit is $p_{\theta}(0) r-c$. Proposition 1 characterizes the first-best level of quality improvement efforts (note that all the proofs are in the Appendix).

Proposition 1. Under the first-best outcome, the integrated firm invests in quality improvement efforts, i.e., $e_{\theta}^{f b}=1$, if and only if $C_{q} \leq\left[p_{\theta}(1)-p_{\theta}(0)\right] r$.

In case of Proposition 1, the integrated firm internalizes both the costs and benefits of the quality improvement efforts in the supply chain. The cost of improvement efforts is simply $C_{q}$. The benefits come from an increase in the likelihood of the quality of the end product conforming to the specifications, owing to quality improvement efforts made at source (which is assumed to be equal to $\left.p_{\theta}(1)-p_{\theta}(0)\right)$ multiplied by the loss of unit profit opportunity avoided, $r$. To sum up, as long as the $\operatorname{cost} C_{q}$ is less than the (expected) benefits $\left[p_{\theta}(1)-p_{\theta}(0)\right] r$, the integrated firm invests in quality improvement efforts. Otherwise, it would not, i.e., $e_{\theta}=1$ if and only if $C_{q} \leq\left[p_{\theta}(1)-p_{\theta}(0)\right] r$.

In the following two sections, we address the first research question in $\S 1$ by characterizing the optimal QE and QS contracts under information asymmetry and benchmark them against the firstbest outcome, in order to evaluate the impact of the optimal QE and QS contracts on the payoffs of the individual supply chain parties as well as the supply chain as a whole.

\section{The Quality-at-the-End (QE) Contract}

In this section, we characterize the optimal QE contract under information asymmetry. As discussed, after production is complete, the manufacturer decides whether to perform a quality inspection test $(T=1)$ or not $(T=0)$. If the manufacturer decides not to test the production outcome $(T=0)$, she has to make the contingent payment to the supplier unconditionally. However, if the manufacturer chooses to inspect the production outcome $(T=1)$, she incurs $\psi_{t}$, and the $\theta$-type supplier receives the contingent payment $Y_{\theta}$ if and only if the production outcomes pass the test, i.e., $\tilde{q}_{\theta}=1$. We do 
consider the fact that the test is not entirely accurate and therefore denote $\mu_{t}$ as the degree of "inaccuracy" of the test. More specifically, $\mu_{t}$ is defined as the conditional probability of the end product passing the inspection test even though it is defective, i.e., $\mu_{t}=\operatorname{Pr}\left[\right.$ test report $=$ non-defective $\mid \tilde{q}_{\theta}=$ 0]. Since the manufacturer does not observe the supplier's quality improvement efforts, the resulting interaction between the manufacturer and the supplier can be modelled as a simultaneous game known as an "inspection game" (see Babich and Tang (2012)). Table 1 presents the strategy of the quality inspection game, where the first expression in each cell is the expected profit of the supplier, and the second expression is the expected profit of the manufacturer.

Table 1 The supplier's (top expression) and manufacturer's (bottom expression) expected profits in the quality inspection game

\begin{tabular}{cccc}
\hline $\begin{array}{c}\text { Supplier's } \\
\text { effort }\end{array}$ & & \multicolumn{2}{c}{ Quality inspection test } \\
$e_{\theta}=1$ & & $\frac{p_{\theta}(1) r-\omega_{\theta}-\left[p_{\theta}(1)+\mu_{t}\left(1-p_{\theta}(1)\right)\right] Y_{\theta}-\psi_{t}}{\omega_{\theta}-c-C_{q}+\left[p_{\theta}(1)+\mu_{t}\left(1-p_{\theta}(1)\right)\right] Y_{\theta}}$ & $\frac{p_{\theta}(1) r-\omega_{\theta}-Y_{\theta}}{\omega_{\theta}-c-C_{q}+Y_{\theta}}$ \\
\cline { 1 - 1 }$e_{\theta}=0$ & $\omega_{\theta}-c+\left[p_{\theta}(0)+\mu_{t}\left(1-p_{\theta}(0)\right)\right] Y_{\theta}$ & & $\omega_{\theta}-c+Y_{\theta}$ \\
& $p_{\theta}(0) r-\omega_{\theta}-\left[p_{\theta}(0)+\mu_{t}\left(1-p_{\theta}(0)\right)\right] Y_{\theta}-\psi_{t}$ & & $p_{\theta}(0) r-\omega_{\theta}-Y_{\theta}$ \\
\hline
\end{tabular}

We analyze the optimal QE contract through three steps. Following backward induction, first, we start with characterizing all the possible Nash equilibria of the inspection game in $\S 4.1$. Then, in $\S 4.2$, we present the optimal QE contract that would induce each equilibrium in the inspection game. Finally, by comparing the information rent and channel loss incurred under each contract, we characterize the optimal QE contract from the manufacturer's perspective.

\subsection{Analysis of the Quality Inspection Game}

Let $\alpha_{\theta}$ and $\beta_{\theta}$ denote the probabilities associated with the manufacturer conducting a quality inspection test $(T=1)$ and the $\theta$-type supplier making process improvement efforts $\left(e_{\theta}=1\right)$, respectively. Given the QE contract terms $\left(\omega_{\theta}, Y_{\theta}\right)$, Lemma 1 below characterizes all the possible Nash equilibria of the inspection game:

Lemma 1. Given a $Q E$ contract $\left(\omega_{\theta}, Y_{\theta}\right)$ offered by the manufacturer, the Nash equilibria of the quality inspection game are characterized for all the regions as follows:

- Region I: There is a pure strategy Nash equilibrium under which $\alpha_{\theta}=1$ and $\beta_{\theta}=1$ if $\psi_{t} \leq$ $\left(1-\mu_{t}\right)\left[1-p_{\theta}(1)\right] Y_{\theta}$ and $C_{q} \leq\left(1-\mu_{t}\right)\left[p_{\theta}(1)-p_{\theta}(0)\right] Y_{\theta}$.

- Region II: there is a mixed strategy Nash equilibrium under which $\alpha_{\theta}=\frac{C_{q}}{\left(1-\mu_{t}\right)\left[p_{\theta}(1)-p_{\theta}(0)\right] Y_{\theta}}$, and $-\beta_{\theta}=1$ if $\left(1-\mu_{t}\right)\left[1-p_{\theta}(1)\right] Y_{\theta}=\psi_{t}$.

$-\beta_{\theta}=\frac{\left(1-\mu_{t}\right)\left[1-p_{\theta}(0)\right] Y_{\theta}-\psi_{t}}{\left(1-\mu_{t}\right)\left[p_{\theta}(1)-p_{\theta}(0)\right] Y_{\theta}}$ if $\left(1-\mu_{t}\right)\left[1-p_{\theta}(1)\right] Y_{\theta}<\psi_{t} \leq\left(1-\mu_{t}\right)\left[1-p_{\theta}(0)\right] Y_{\theta}$ and $C_{q} \leq(1-$ $\left.\mu_{t}\right)\left[p_{\theta}(1)-p_{\theta}(0)\right] Y_{\theta}$. 
- Region III: There is a pure strategy Nash equilibrium under which $\alpha_{\theta}=1$ and $\beta_{\theta}=0$ if $\psi_{t} \leq$ $\left(1-\mu_{t}\right)\left[1-p_{\theta}(0)\right] Y_{\theta}$ and $C_{q}>\left(1-\mu_{t}\right)\left[p_{\theta}(1)-p_{\theta}(0)\right] Y_{\theta}$.

- Region IV: there is a pure strategy Nash equilibrium under which $\alpha_{\theta}=0$ and $\beta_{\theta}=0$ if $\psi_{t}>$ $\left(1-\mu_{t}\right)\left[1-p_{\theta}(0)\right] Y_{\theta}$.

Based on Lemma 1, our main observations are as follows: First, if the cost of inspection $\psi_{t}$ and cost of quality improvement efforts $C_{q}$ are sufficiently low (Region I), the supplier makes quality improvement efforts and the manufacturer inspects the quality of the end product. As the cost of inspection $\psi_{t}$ increases (while keeping $C_{q}$ constant), the manufacturer first starts employing a mixed strategy in Region II in order to lower the expected cost of the inspection, and then completely stops the inspection in Region IV. Anticipating this, the supplier also employs a mixed strategy equilibrium in Region II and completely stops making quality improvement efforts in Region IV. As we will see later, this leads to a deviation from the first-best outcome, causing a channel loss for the supply chain.

\subsection{The Optimal QE Contract}

After we characterize all the Nash equilibria of the inspection sub-game, the next step is to find the optimal QE contract that would induce each Nash equilibrium. Let $\alpha_{\theta}$ and $\beta_{\theta}$ denote one of the Nash equilibria characterized in Lemma 1. Then, the optimal QE contract that induces $\alpha_{\theta}$ and $\beta_{\theta}$ can be obtained by solving the following mathematical program:

$$
\begin{aligned}
\max _{\left(\omega_{h} \geq 0, Y_{h} \geq 0\right),\left(\omega_{l} \geq 0, Y_{l} \geq 0\right)} & \nu \pi_{h}^{M}\left(\omega_{h}, Y_{h} \mid \alpha_{h}, \beta_{h}\right)+(1-\nu) \pi_{l}^{M}\left(\omega_{l}, Y_{l} \mid \alpha_{l}, \beta_{l}\right) \\
& \pi_{h}^{S}\left(\omega_{h}, Y_{h} \mid \alpha_{h}, \beta_{h}\right) \geq 0 \\
& \pi_{l}^{S}\left(\omega_{l}, Y_{l} \mid \alpha_{l}, \beta_{l}\right) \geq 0 \\
& \pi_{h}^{S}\left(\omega_{h}, Y_{h} \mid \alpha_{h}, \beta_{h}\right) \geq \pi_{h}^{S}\left(\omega_{l}, Y_{l} \mid \alpha_{l}, \tilde{\beta}_{h}\right) \\
& \pi_{l}^{S}\left(\omega_{l}, Y_{l} \mid \alpha_{l}, \beta_{l}\right) \geq \pi_{l}^{S}\left(\omega_{h}, Y_{h} \mid \alpha_{h}, \tilde{\beta}_{l}\right) \\
& \tilde{\beta}_{\theta}=\underset{\beta_{\theta} \in[0,1]}{\arg \max } \pi_{\theta}^{S}\left(\omega_{\check{\theta}}, Y_{\check{\theta}} \mid \alpha_{\check{\theta}}, \beta_{\theta}\right), \check{\theta} \neq \theta
\end{aligned}
$$

where $\pi_{\theta}^{M}\left(\omega_{\theta}, Y_{\theta} \mid \alpha_{\theta}, \beta_{\theta}\right)$ and $\pi_{\theta}^{S}\left(\omega_{\theta}, Y_{\theta} \mid \alpha_{\theta}, \beta_{\theta}\right)$ represent the manufacturer's and the supplier's expected profits, given that the manufacturer offers a menu of contracts $\left(\omega_{\theta}, Y_{\theta}\right)$ and, subsequently, the manufacturer and the supplier conduct an equilibrium inspection $\alpha_{\theta}$ and make quality improvement efforts $\beta_{\theta}$, respectively. The objective function (2) is formed by taking the expectation of the manufacturer's profit expressions with respect to a-priori beliefs for $h$ - and $l$-type suppliers, i.e., $\nu$ and $1-\nu$, respectively. Individual rationality (IR) constraints $(3-4)$ ensure a non-zero profit for the $\theta$-type supplier, while incentive compatibility (IC) constraints $(5-6)$ ensure that each supplier type self-selects the contract designed for him. Finally, the moral hazard (MH) constraint (7) defines the 
optimal effort for the $\theta$-type supplier, should he mimic the $\check{\theta}$-type supplier (off-equilibrium decision), where $\theta \neq \check{\theta}$.

At the last stage of our analysis, we compare the optimal QE contracts that are implementable and identify the one that maximizes the manufacturer's expected profit. In order to do so, we need to analyze the factors that contribute to channel loss and information rent.

We begin by considering channel loss. There are two types of channel loss: The first is directly incurred by the manufacturer who conducts a quality inspection with probability $\alpha_{\theta}$. The second is indirectly caused by the supplier who does not make quality improvement efforts. Since there are two types of suppliers, each of whom can potentially cause a loss of $\left(p_{\theta}(1)-p_{\theta}(0)\right) r-C_{q}$ due to insufficient or absent efforts with probability $1-\beta_{\theta}$, the total channel loss incurred by the manufacturer can be expressed as

$$
\text { Channel loss under } \mathrm{QE}=\underbrace{\sum_{\theta=l, h} \nu_{\theta} \alpha_{\theta} \psi_{t}}_{\text {Direct cost }}+\underbrace{\sum_{\theta=l, h} \nu_{\theta}\left(1-\beta_{\theta}\right)\left[\left(p_{\theta}(1)-p_{\theta}(0)\right) r-C_{q}\right]}_{\text {Indirect cost }}
$$

where $\nu_{h}=\nu$ and $\nu_{l}=1-\nu$. Next, we consider information rent. Note that the information rent under the optimal QE contract is paid only to the $h$-type supplier. This is because, in an equilibrium, it is only the $h$-type supplier that has the incentive to deviate. Therefore, in order to satisfy the IC constraint of the $h$-type supplier, the manufacturer has to pay a net amount, which the supplier would earn if he deviates and mimics the $l$-type. There are two types of information rent that the $h$-type supplier would potentially ask for. The first type, denoted by the term "moral hazard", is paid when $h$ - and $l$-types make different quality improvement efforts, i.e., $\beta_{h} \neq \beta_{l}$. The second type, denoted by the term "reliability-asymmetry", is paid due to the fact that $l$ - and $h$-type suppliers are inherently different in their degrees of reliability. Note that this second type is paid even if $h$ - and $l$-type suppliers make the same quality improvement efforts, i.e., $\beta_{h}=\beta_{l}$. Adding these two terms, we obtain the following expression for the information rent paid to the $h$-type supplier:

$$
\text { Information rent under } \mathrm{QE}=\underbrace{\left(\beta_{l}-\beta_{h}\right) C_{q}}_{\text {Moral hazard term }}+\underbrace{\left(1-\mu_{t}\right) \alpha_{l}\left[\beta_{h} p_{h}(1)-\beta_{l} p_{l}(1)\right]^{+} Y_{l}}_{\text {reliability-asymmetry term }}
$$

By comparing the information rent and channel loss incurred in each contracting strategy, we are able to characterize the optimal QE contract.

Proposition 2. The optimal $Q E$ contracts $\left(\omega_{\theta}, Y_{\theta}\right)$ and the decomposition of total inefficiency (into information rent and channel loss) resulting from their implementations are characterized in Table 2. Also, the Figure provided in Table 2 shows the regions for each contract as well as the Nash equilibrium induced for the subsequent inspection sub-game. 
Nikoofal and Gümüş: Quality at the Source or at the End?

Manufacturing \& Service Operations Management 00(0), pp. 000-000, (C) 0000 INFORMS

Table 2 Contract terms, equilibrium decisions, information rent, and channel loss under the optimal QE contract

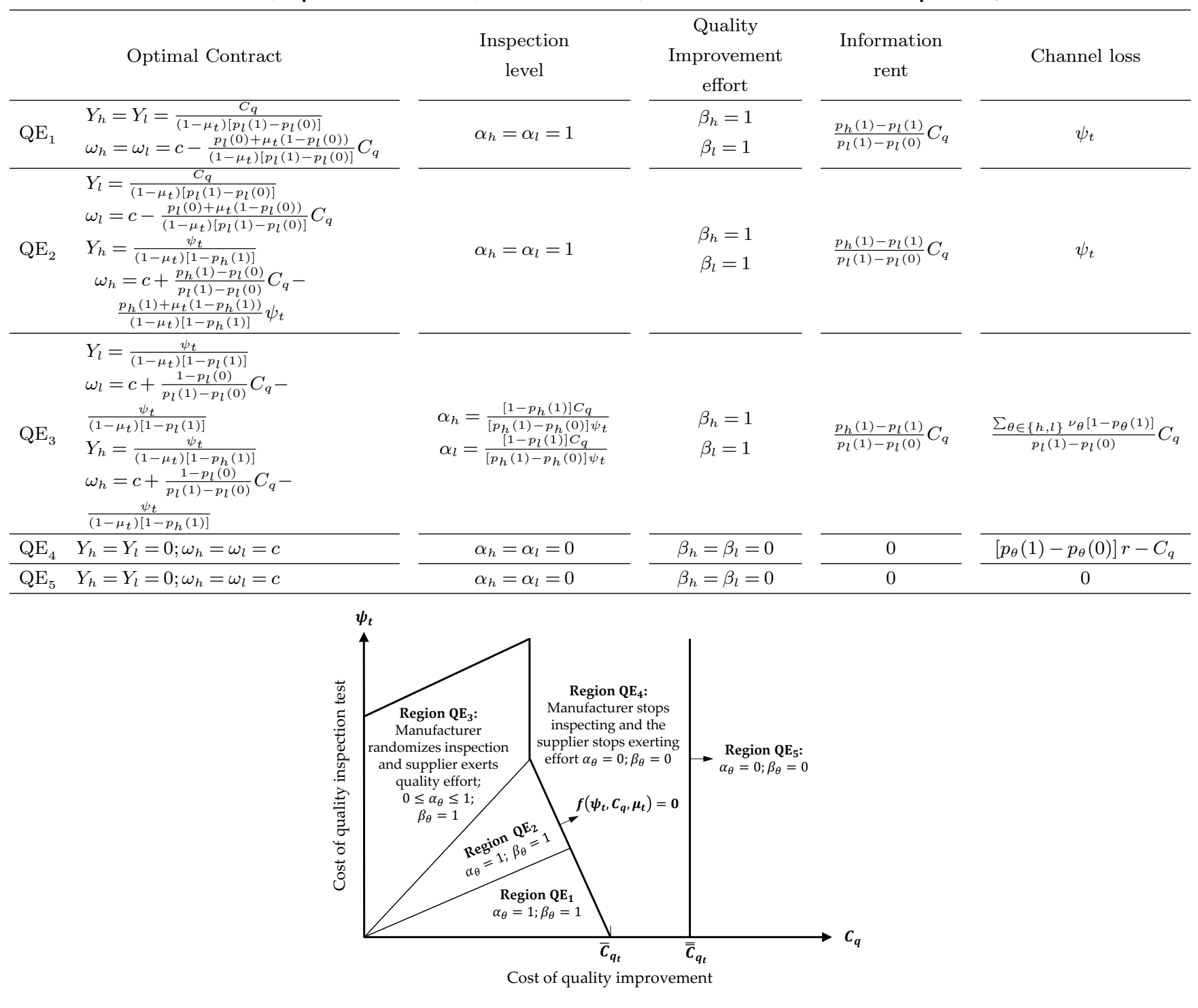

Notes: The information rent is paid only to the $h$-type supplier (with probability $\nu$ ). Expressions for $f\left(\psi_{t}, C_{q}, \mu_{t}\right), \bar{C}_{q_{t}}$, and $\overline{\bar{C}}_{q_{t}}$ are provided in the Appendix.

First, recall that it is never optimal to make quality improvement efforts in the first-best outcome when $C_{q} \geq\left[p_{l}(1)-p_{l}(0)\right] r$. Hence, under information asymmetry, when this condition is satisfied, the manufacturer would offer a $\mathrm{QE}$ contract, denoted by $Q E_{5}$ in Table 2, which would induce $\beta_{h}=\beta_{l}=0$ in equilibrium. Second, Lemma 1 shows that even though there exists a pure strategy Nash equilibrium (Region III) wherein the manufacturer always conducts an inspection and the supplier never makes quality improvement efforts, according to Proposition 2 above, it is never optimal for the manufacturer to induce pure strategy equilibrium (Region III). This is because the Nash equilibrium of Region III is always dominated by the Nash equilibrium of Region IV in which neither does the manufacturer conduct an inspection and nor does the supplier make quality 
improvement efforts. Therefore, the optimal contract is ultimately determined by comparing the optimal QE contracts that would induce Nash equilibria in Regions I, II, and IV, where the first two correspond to a pure and mixed strategy equilibria of inducing the supplier to make process improvement efforts and the third corresponds to not inducing the supplier to make any effort.

In Regions I and II, the manufacturer incurs a direct channel loss, which increases with the cost of inspection $\psi_{t}$ and the cost of quality improvement $C_{q}$, while in Region IV, the manufacturer incurs an indirect channel loss when the supplier does not make an effort, which decreases in $C_{q}$. In addition, in Regions I and II, the manufacturer also incurs information rent. Note that since both $h$ - and $l$-type suppliers make the same quality improvement efforts, i.e., $e_{h}=e_{l}=1$, the first term of information rent in Eq. (9) vanishes. The second term is determined by the contingent payment received by the $l$-type supplier, i.e., $Y_{l}$. Since the $l$-type supplier is induced to make a quality improvement effort - which means his quality improvement $\operatorname{cost} C_{q}$ is subsidized by the manufacturer as a contingent payment - it implies that the information rent received by $h$-type increases in $C_{q}$. To summarize, the total agency cost incurred under the optimal contract for Regions I and II increases in $\psi_{t}$ and $C_{q}$, while that for Region IV decreases in $C_{q}$. This implies that if both $C_{q}$ and $\psi_{t}$ are sufficiently low, the manufacturer offers the optimal QE contract, denoted by $Q E_{1}$, $Q E_{2}$ and $Q E_{3}$ in Table 2, which would induce $\beta_{\theta}=1$ for both $\theta=\{h, l\}$. Otherwise, she would offer the optimal QE contract, denoted by $Q E_{4}$ in Table 2 , which would induce $\beta_{\theta}=0$ for both $\theta=\{h, l\}$.

Finally, we note that the threshold $f\left(\psi_{t}, C_{q}, \mu_{t}\right)$ decreases in $\mu_{t}$ for a given $\psi_{t}$ and $C_{q}$ (refer to the Appendix for the proof). This implies that ceteris paribus the regions under which the $\theta$-type supplier is induced to make quality improvement efforts decreases in $\mu_{t}$. The rationale behind this is related to the degree of precision gauged from the inspection. The less accurate the inspection outcome (corresponding to a higher $\mu_{t}$ ), the harder it becomes for the manufacturer to incentivize the supplier's efforts based on a noisy signal. Hence, when the degree of inaccuracy of the inspection test $\mu_{t}$ exceeds the threshold $\bar{\mu}_{t}\left(C_{q}, \psi_{t}\right)$ implied by $f\left(\psi_{t}, C_{q}, \mu_{t}\right) \leq 0$, the quality improvement effort simply ceases to be implementable. Note that the impact of $\mu_{t}$ on the quality improvement effort comes directly from the implementability constraints of the optimal QE contract rather than the comparison between its agency costs, as the latter is used to evaluate the impacts of $\psi_{t}$ and $C_{q}$ above.

\section{The Quality-at-the-Source (QS) Contract}

In this section, we characterize the optimal QS contract under information asymmetry. Similar to the QE contract, this too involves two stages. In the first stage, the manufacturer designs a menu of contracts $\left(\omega_{\theta}, Y_{\theta}, E_{\theta}\right)$ for the supplier. In the second stage, denoted by "audit game", the supplier decides whether to make quality improvement efforts and the manufacturer decides whether to 
audit the supplier's efforts. We denote the cost of audit incurred by the manufacturer by $\psi_{a}$ and the manufacturer's auditing decision by $A$, where $A=1$ and $A=0$ correspond to "audit" and "no-audit", respectively. The contingent term $Y_{\theta}$ in the QS contract is paid to the supplier if his quality improvement decision $e_{\theta}$ matches the level of effort $E_{\theta}$ required by the contract, given that the manufacturer decides to conduct an audit, i.e., $A=1$. If the manufacturer does not conduct an audit, i.e., $A=0$, the contingent term $Y_{\theta}$ is paid to the supplier irrespective of whether he made quality improvement efforts. Similar to inspection, we also consider that an audit is not accurate and we thus model the degree of inaccuracy with $\mu_{a}$, where $\mu_{a}$ denotes the conditional probability of an audit, indicating that process improvement efforts have been made by the supplier when indeed they have not, i.e., $\mu_{a}=\operatorname{Pr}\left[\right.$ a process improvement effort is made based on the audit $\left.\mid e_{\theta}=0\right]$. Table 3 presents the strategic form of the audit game, where the first expression in each cell is the expected profit of the supplier, and the second expression is the expected profit of the manufacturer.

Table 3 The Supplier's (First Expression) and Manufacturer's (Second Expression) Expected Profits Under the

\begin{tabular}{|c|c|c|}
\hline \multicolumn{3}{|c|}{ Audit Game } \\
\hline Supplier's & \multicolumn{2}{|c|}{ Effort inspection level $\left(E_{\theta}=1\right)$} \\
\hline effort & $A=1$ & $A=0$ \\
\hline$e_{\theta}=1$ & $\omega_{\theta}+Y_{\theta}-c-C_{q}, p_{\theta}(1) r-\omega_{\theta}-Y_{\theta}-\psi_{a}$ & $\omega_{\theta}+Y_{\theta}-c-C_{q}, p_{\theta}(1) r-\omega_{\theta}-Y_{\theta}$ \\
\hline$e_{\theta}=0$ & $\omega_{\theta}-c+\mu_{a} Y_{\theta}, p_{\theta}(0) r-\omega_{\theta}-\mu_{a} Y_{\theta}-\psi_{a}$ & $\omega_{\theta}+Y_{\theta}-c, p_{\theta}(0) r-\omega_{\theta}-Y_{\theta}$ \\
\hline Supplier's & \multicolumn{2}{|c|}{ Effort inspection level $\left(E_{\theta}=0\right)$} \\
\hline effort & $A=1$ & $A=0$ \\
\hline$e_{\theta}=1$ & $\omega_{\theta}-c-C_{q}, p_{\theta}(1) r-\omega_{\theta}-\psi_{a}$ & $\omega_{\theta}+Y_{\theta}-c-C_{q}, p_{\theta}(1) r-\omega_{\theta}-Y_{\theta}$ \\
\hline$e_{\theta}=0$ & $\omega_{\theta}+Y_{\theta}-c, p_{\theta}(0) r-\omega_{\theta}-Y_{\theta}-\psi_{a}$ & $\omega_{\theta}+Y_{\theta}-c, p_{\theta}(0) r-\omega_{\theta}-Y_{\theta}$ \\
\hline
\end{tabular}

Similar to the QE contract, we follow three steps to characterize the optimal QS contract. Following backward induction, first, we start with characterizing all the possible Nash equilibria of the audit game. Then, we obtain the optimal QS contract that would induce each equilibrium in the audit game. Finally, comparing the information rent and channel loss incurred under each QS contract, we characterize the optimal QS contract.

\subsection{Analysis of the Audit Game}

There are two matrix games in Table 4 , one for $E_{\theta}=0$ and the other for $E_{\theta}=1$. It is easy to verify that there is always a unique pure Nash equilibrium for $E_{\theta}=0$, where $A=e_{\theta}=0$, i.e., neither does the manufacturer conduct an audit, nor does the supplier make quality improvement efforts. Therefore, in what follows, we restrict our attention to the case where the manufacturer sets $E_{\theta}=1$. Considering the mixed strategies, we define $\gamma_{\theta}$ and $\beta_{\theta}$ to denote the probabilities of the manufacturer conducting an audit and the $\theta$-type supplier making quality improvement efforts, 
respectively. Multiplying these probabilities with the players' profits in Table 4 for $E_{\theta}=1$, the manufacturer's expected profit function is given by

$$
\pi_{\theta}^{M}\left(\omega_{\theta}, Y_{\theta} \mid \gamma_{\theta}, \beta_{\theta}\right)=\left[\beta_{\theta} p_{\theta}(1)+\left(1-\beta_{\theta}\right) p_{\theta}(0)\right] r-\omega_{\theta}-Y_{\theta}+\gamma_{\theta}\left[\left(1-\mu_{a}\right)\left(1-\beta_{\theta}\right) Y_{\theta}-\psi_{a}\right]
$$

Similarly, the $\theta$-type supplier's profit function can be expressed as follows:

$$
\pi_{\theta}^{S}\left(\omega_{\theta}, Y_{\theta} \mid \gamma_{\theta}, \beta_{\theta}\right)=\omega_{\theta}-c+\left[1-\gamma_{\theta}\left(1-\mu_{a}\right)\right] Y_{\theta}+\beta_{\theta}\left[\gamma_{\theta}\left(1-\mu_{a}\right) Y_{\theta}-C_{q}\right]
$$

The following Lemma 2 presents the solution of the audit game presented in Table 3.

Lemma 2. Given a $Q S$ contract $\left(\omega_{\theta}, Y_{\theta}, E_{\theta}\right)$ offered by the manufacturer, the Nash equilibria of the audit game are as follows:

- If the manufacturer sets $E_{\theta}=0$, then the unique Nash equilibrium is $\gamma_{h}=\gamma_{l}=0$ and $\beta_{\theta}=0$.

- If the manufacturer sets $E_{\theta}=1$, then, there are three regions under which a unique Nash equilibrium is characterized as follows:

-Region I: There is a mixed strategy Nash equilibrium under which $\gamma_{\theta}=\frac{C_{q}}{\left(1-\mu_{a}\right) Y_{\theta}}$ and $\beta_{\theta}=$ $1-\frac{\psi_{a}}{\left(1-\mu_{a}\right) Y_{\theta}}$ if $\psi_{a} \leq\left(1-\mu_{a}\right) Y_{\theta}$ and $C_{q} \leq\left(1-\mu_{a}\right) Y_{\theta}$.

- Region II: There is a pure strategy Nash equilibrium under which $\gamma_{\theta}=1$ and $\beta_{\theta}=0$ if $\psi_{a}<$ $\left(1-\mu_{a}\right) Y_{\theta}$ and $C_{q}>\left(1-\mu_{a}\right) Y_{\theta}$.

-Region III: There is a pure strategy Nash equilibrium under which $\gamma_{\theta}=0$ and $\beta_{\theta}=0$ if $\psi_{a}>\left(1-\mu_{a}\right) Y_{\theta}$.

As opposed to the inspection game analyzed as per the QE contract, the audit game analyzed as per the QS contract never yields a pure strategy equilibrium according to which the manufacturer always conducts an audit and the supplier always makes quality improvement efforts. The rationale behind this is related to the increased risk shouldered by the manufacturer as per the QS contract compared to the QE contract. Under the QE contract, an inspection provides the manufacturer with information regarding the quality of the end product, while under the QS contract, an audit validates only the supplier's efforts. This means that upon conducting an audit, the manufacturer may end up making the contingent payment and at the same time receiving a product that does not satisfy the end-quality. Therefore, the audit mechanism as per the QS contract is more expensive than as per the QE contract in terms of inducing the supplier's quality improvement efforts. Hence, in Region I, the manufacturer reduces the cost by auditing only occasionally with probability $\gamma_{\theta}=\frac{C_{q}}{\left(1-\mu_{a}\right) Y_{\theta}}$, which also leads to the $\theta$-type supplier making quality improvement efforts only occasionally with probability $\beta_{\theta}=1-\frac{\psi_{a}}{\left(1-\mu_{a}\right) Y_{\theta}}$. Moreover, auditing is mainly used by the manufacturer as an inspection mechanism to avoid paying the supplier when he does not make the required effort. Because of this, an increase in contingent payment $Y_{\theta}$ increases the likelihood of the $\theta$-type supplier making 
quality improvement efforts, which in turn decreases the need for the manufacturer to conduct an audit. Finally, as $\psi_{a}$ increases, it becomes more expensive for the manufacturer to conduct an audit. In response to this, the likelihood of the supplier making quality improvement efforts also lowers. Hence, when $\psi_{a}$ is sufficiently high, i.e., in Region III, neither does the manufacturer conduct an audit nor does the supplier make efforts.

\subsection{The Optimal QS Contract}

Given the equilibrium characterization of the audit game, the optimal QS contract can be obtained by solving the following mathematical program:

$$
\begin{aligned}
\max _{\left(\omega_{h} \geq 0, Y_{h} \geq 0, E_{h}\right),\left(\omega_{l} \geq 0, Y_{l} \geq 0, E_{l}\right)} & \nu \pi_{h}^{M}\left(\omega_{h}, Y_{h} \mid \gamma_{h}, \beta_{h}\right)+(1-\nu) \pi_{l}^{M}\left(\omega_{l}, Y_{l} \mid \gamma_{l}, \beta_{l}\right) \\
& \pi_{h}^{S}\left(\omega_{h}, Y_{h} \mid \gamma_{h}, \beta_{h}\right) \geq 0 \\
& \pi_{l}^{S}\left(\omega_{l}, Y_{l} \mid \gamma_{l}, \beta_{l}\right) \geq 0 \\
& \pi_{h}^{S}\left(\omega_{h}, Y_{h} \mid \gamma_{h}, \beta_{h}\right) \geq \pi_{h}^{S}\left(\omega_{l}, Y_{l} \mid \gamma_{l}, \tilde{\beta}_{h}\right) \\
& \pi_{l}^{S}\left(\omega_{l}, Y_{l} \mid \gamma_{l}, \beta_{l}\right) \geq \pi_{l}^{S}\left(\omega_{h}, Y_{h} \mid \gamma_{h}, \tilde{\beta}_{l}\right) \\
& \tilde{\beta}_{\theta}=\underset{\beta_{\theta} \in[0,1]}{\arg \max } \pi_{\theta}^{S}\left(\omega_{\check{\theta}}, Y_{\check{\theta}} \mid \gamma_{\check{\theta}}, \beta_{\theta}\right), \check{\theta} \neq \theta
\end{aligned}
$$

where the objective is to maximize the manufacturer's expected profit $\pi_{\theta}^{M}\left(\omega_{\theta}, Y_{\theta} \mid \gamma_{\theta}, \beta_{\theta}\right)$ with respect to $\theta=h$ and $\theta=l$. Constraints $(13-14)$ and $(15-16)$ are, respectively, IR and IC constraints for $h$ - and $l$-type suppliers, and constraint (17) defines the optimal level of effort for the $\theta$-type supplier, should he mimic the $\check{\theta}$-type supplier (off-equilibrium decision), where $\check{\theta} \neq \theta$.

In order to characterize the optimal QS contract, we first need to determine the optimal solution of the manufacturer's contract design problem $(12-17)$ for each of the Nash equilibria characterized in Lemma 2. We can then determine the optimal QS contract by comparing the total inefficiencies incurred under each region. Delegating the details to the Appendix, we provide the complete characterization in the following Proposition:

Proposition 3. The optimal QS contract $\left(\omega_{\theta}, Y_{\theta}\right)$, the Nash equilibrium induced under the optimal contract, and the total inefficiency resulting from the equilibrium are provided in Table 4.

First of all, the equilibrium depends on whether the product cost is low (i.e., $c \leq\left[p_{\theta}(1)-p_{\theta}(0)\right] r$ ) or high (i.e., $\left.c>\left[p_{\theta}(1)-p_{\theta}(0)\right] r\right)$. However, in both cases, when $C_{q}>\left[p_{\theta}(1)-p_{\theta}(0)\right] r$, making quality improvement efforts becomes too expensive for the manufacturer. Hence, when this condition is satisfied, the manufacturer offers an optimal QS contract, denoted by $Q S_{3}$ in Table 4, which would induce $\beta_{\theta}=0$ for both $\theta=h$ and $\theta=l$. Therefore, we restrict our attention to the case where $C_{q} \leq\left[p_{\theta}(1)-p_{\theta}(0)\right] r$. Recall from Lemma 2 that there are three equilibria that can be induced under the optimal QS contract. However, as shown in the proof of Proposition 3, we can always eliminate 
Table 4 Contract terms, equilibrium decisions, and channel loss under the optimal QS contract

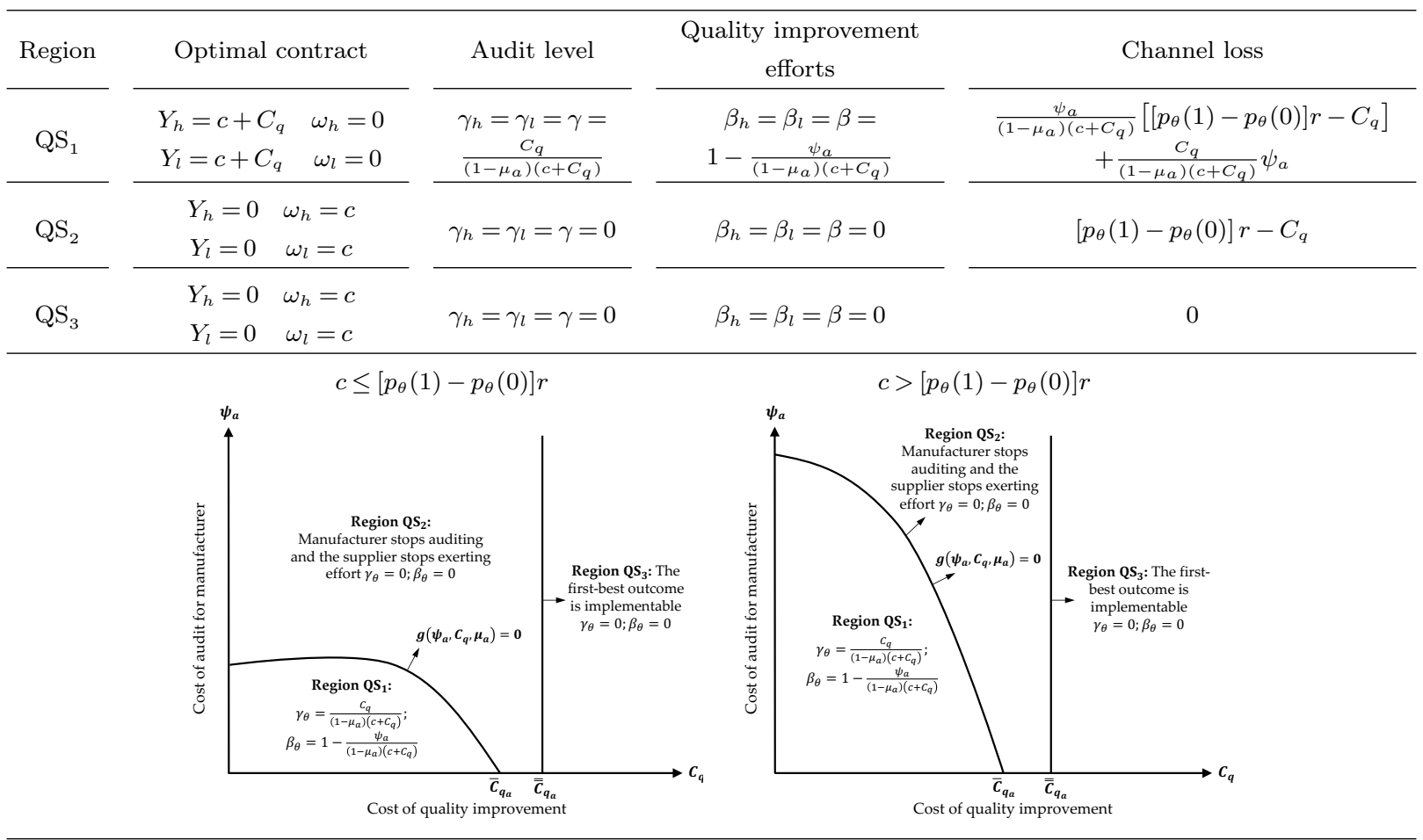

Notes. $\overline{\bar{C}}_{q_{a}}=\left[p_{\theta}(1)-p_{\theta}(0)\right] r$. The expressions for $g\left(\psi_{a}, C_{q}, \mu_{a}\right)$, and $\bar{C}_{q_{a}}$ are provided in the Appendix.

the pure equilibrium in Region II in Lemma 2 above, in which the manufacturer always conducts an audit and the supplier never makes quality improvement efforts, because this equilibrium is dominated by those characterized in Regions I and III. Note that in the mixed equilibrium of Region I, the manufacturer conducts an inspection and the supplier makes quality improvement efforts with the probabilities $\gamma_{\theta}$ and $\beta_{\theta}$, respectively. However, in the pure equilibrium of Region III, neither does the manufacturer conduct an inspection nor does the supplier make quality improvement efforts. This means that the optimal QS contract is determined by comparing the total inefficiencies incurred by the manufacturer under Regions I and III. That said, as opposed to the optimal QE contract, the only inefficiency incurred under the optimal QS contract is channel loss. This relates to the fact that the optimal contract is always of the pooling type for the optimal QS model. Note that under the optimal QS contract, the manufacturer makes the contingent payment based on the effort, while under the optimal QE contract, she pays based on the output. Since the private information, i.e., the difference in reliability between $h$ - and $l$-type suppliers, affects only the output and not the level of effort, the contingent payment under the optimal QS contract turns out to be type-independent, whereas under the optimal QE contract, it is necessarily type-dependent. Hence, under the optimal QS contract, it is sufficient for the manufacturer to offer a pooling contract, which in turn eliminates the need for paying information rent due to the difference in reliability between suppliers. 
Nikoofal and Gümüş: Quality at the Source or at the End?

With regard to channel loss, as in the case of the optimal QE contract, the manufacturer incurs two types of channel losses. The first is directly incurred by the manufacturer who conducts an expensive audit with probability $\gamma$. The second is indirectly caused by a supplier who does not make quality improvement efforts. Since both $h$ - and $l$-type suppliers use the same probability, the total channel loss incurred by the manufacturer can be expressed as

$$
\text { Channel loss under QS }=\underbrace{\gamma \psi_{a}}_{\text {Direct cost }}+\underbrace{(1-\beta)\left[\left(p_{\theta}(1)-p_{\theta}(0)\right) r-C_{q}\right]}_{\text {Indirect cost }}
$$

Finally, in Proposition 3, we identify the optimal QS contracts that induce the equilibria in Regions I and III in Lemma 2 and denote them by $Q S_{1}$ and $Q S_{2}$, respectively, in Table 4 . Note that channel loss incurred under the optimal $Q S_{1}$ contract consists of both direct and indirect costs, whereas under the optimal $Q S_{2}$ contract contains only indirect cost. Analyzing how the direct and indirect costs change with respect to problem parameters yields the optimal QS contract as characterized in Proposition 3.

First, note that the higher the cost of quality improvement $C_{q}$, the more likely it is for the manufacturer to audit the supplier's efforts (refer to probability expression for the audit $\gamma$ ). On the one hand, as the $\gamma$ increases, the direct cost incurred under the $Q S_{1}$ contract also increases. Similarly, the direct cost increases with the cost of the audit $\psi_{a}$. On the other hand, the channel loss incurred under $Q S_{2}$ decreases in $C_{q}$ and does not depend on $\psi_{a}$, because the manufacturer does not incur any direct cost under $Q S_{2}$. Hence, the manufacturer is better off with offering $Q S_{1}$, i.e., inducing the supplier to make quality improvement efforts occasionally, for relatively low values of $C_{q}$ and $\psi_{a}$.

Second, there is a significant difference between the contingent terms paid under the optimal QS and QE contracts. Under the optimal QE contract, the product cost $c$ is always paid upfront, whereas under the optimal QS contract, it is paid contingent to the effort being made by the supplier. This has two consequences: First, as the cost of production $c$ increases, the supplier gets more incentivized to make quality improvement efforts, because he receives the contingent payment only if he does so. Hence, the region under which the supplier makes an effort (i.e., Region $Q S_{1}$ ) grows in $c$ (compare region $Q S_{1}$ for low $c$ to that for high $c$ ). Second, paying the production cost as a contingent term creates a powerful incentive for the supplier to make a concerted quality improvement effort, even if $C_{q}$ becomes relatively high. As a result, the optimal QS contract can induce suppliers to make quality improvement efforts for the values of $C_{q}$ that are relatively higher than those under the optimal QE contract.

Third, similar to the QE contract, the threshold $g\left(\psi_{a}, C_{q}, \mu_{a}\right)$ decreases in $\mu_{a}$ for a given $\psi_{a}$ and $C_{q}$ (refer to the Appendix for the proof). This implies that when the degree of inaccuracy $\mu_{a}$ of the 
audit test exceeds the threshold $\bar{\mu}_{a}$ implied by $\psi_{a} \leq g\left(\psi_{a}, C_{q}, \mu_{a}\right)$, the quality improvement effort ceases to be implementable under the optimal QS contract. The rationale behind this is the same as in the case of the optimal QE contract; hence, we omit the discussion to avoid repetition.

\section{Quality at the Source or Quality at the End?}

In this section, we address the second and third research questions by comparing the optimal QE and QS contracts from the perspectives of the manufacturer, the supplier, as well as the entire supply chain in $§ 6.1$. In order to compare the optimal QE and QS contracts, we need to take into account all sources of inefficiencies, i.e., information rent and channel loss, from the perspectives of the manufacturer, the supplier, and the entire supply chain. In order to control for the effect of cost differences in the comparison, throughout this section, we assume that the costs of inspection and audit are the same ${ }^{2}$, i.e., $\psi_{t}=\psi_{a}=\psi$.

Proposition 4. Suppose $\psi_{t}=\psi_{a}=\psi$. The comparison between optimal $Q E$ and $Q S$ contracts for the manufacturer, the supplier and the entire supply chain is characterized in Table 5 , where $\pi_{Q E}$ and $\pi_{Q S}$ indicate a supply chain entity's profit under the optimal QE and QS contracts, respectively.

First, when both inspection and audit tests are not very accurate, i.e. $\mu_{t}>\bar{\mu}_{t}$ and $\mu_{a}>\bar{\mu}_{a}$, neither QE nor QS contracts would bring any value as the quality improvement efforts cannot be implemented under either of them. Hence, in what follows, we consider $\mu_{t} \leq \bar{\mu}_{t}$ and $\mu_{a} \leq \bar{\mu}_{a}$.

Comparison from the manufacturer's perspective: We need to consider two kinds of losses in a holistic fashion: channel loss and information rent. As mentioned above, channel loss is of two types: direct channel loss is incurred by the manufacturer due to the cost of conducting an inspection, while indirect channel loss is incurred when the supplier does not make quality improvement efforts. The former is influenced by the cost of an inspection or audit, $\psi$, and the latter is determined by how powerful the contract is in incentivizing the supplier to make quality improvement efforts. Therefore, the higher the inspection cost, $\psi$, the higher the direct cost for the manufacturer, while the more powerful the contract, the lower the indirect cost. Furthermore, information rent is affected by the degree of information asymmetry between the manufacturer and the supplier, which in turn is influenced by the difference in the levels of reliability between $h$ - and $l$-type suppliers. Considering these factors, we now compare the optimal QE and QS contracts from the manufacturer's perspective:

With respect to direct channel loss: In general, the optimal QE contract leads to a lower direct cost for the manufacturer compared to the optimal QS contract. This is because when the manufacturer conducts a quality test under the optimal QE contract, it ensures that the output satisfies the

\footnotetext{
${ }^{2}$ For a discussion on the impact of considering different costs in the comparative analyses, please refer to $\S 7.2$.
} 
Table 5 Comparison between the optimal QE and QS contracts

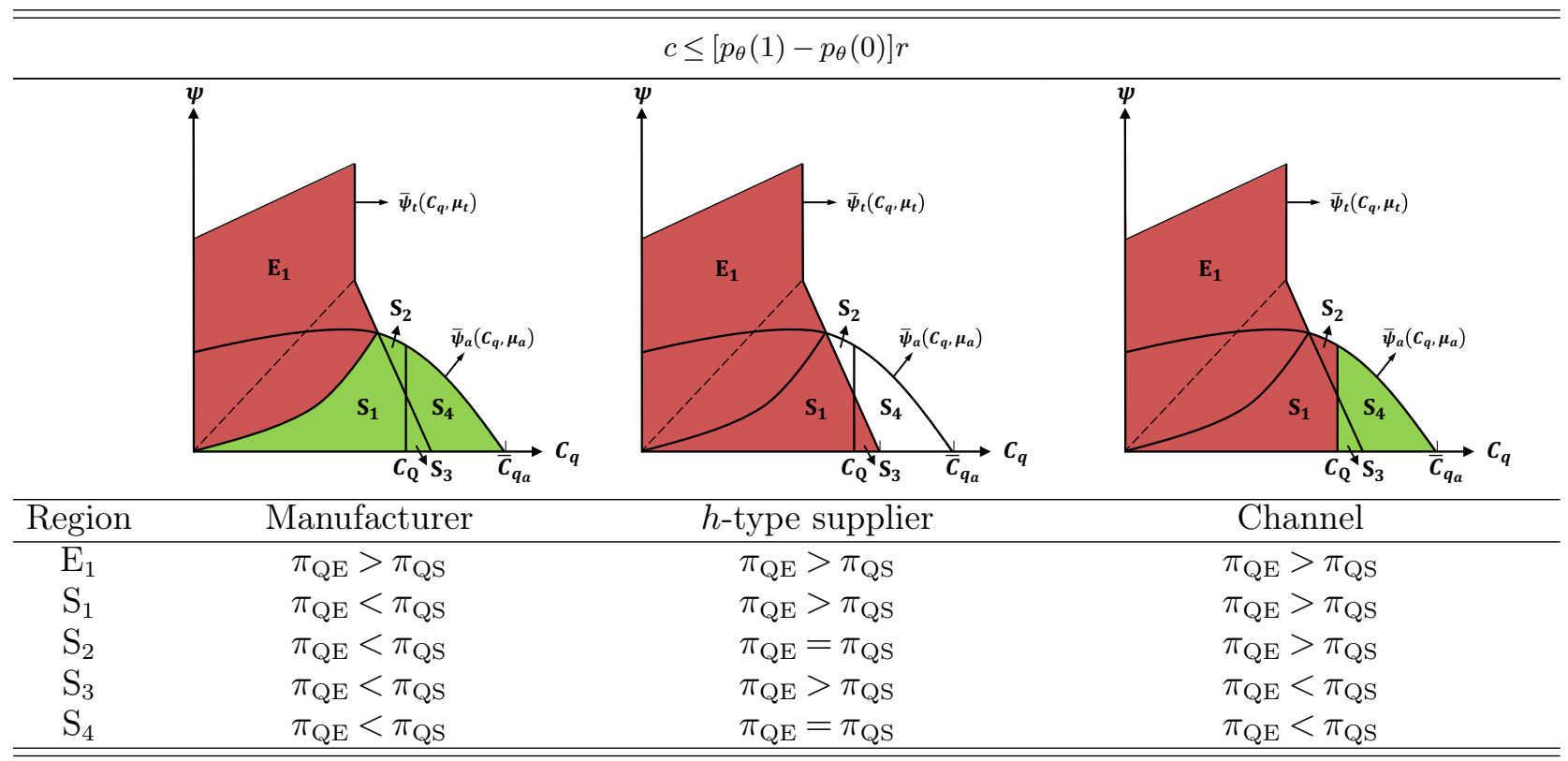

$$
c>\left[p_{\theta}(1)-p_{\theta}(0)\right] r
$$
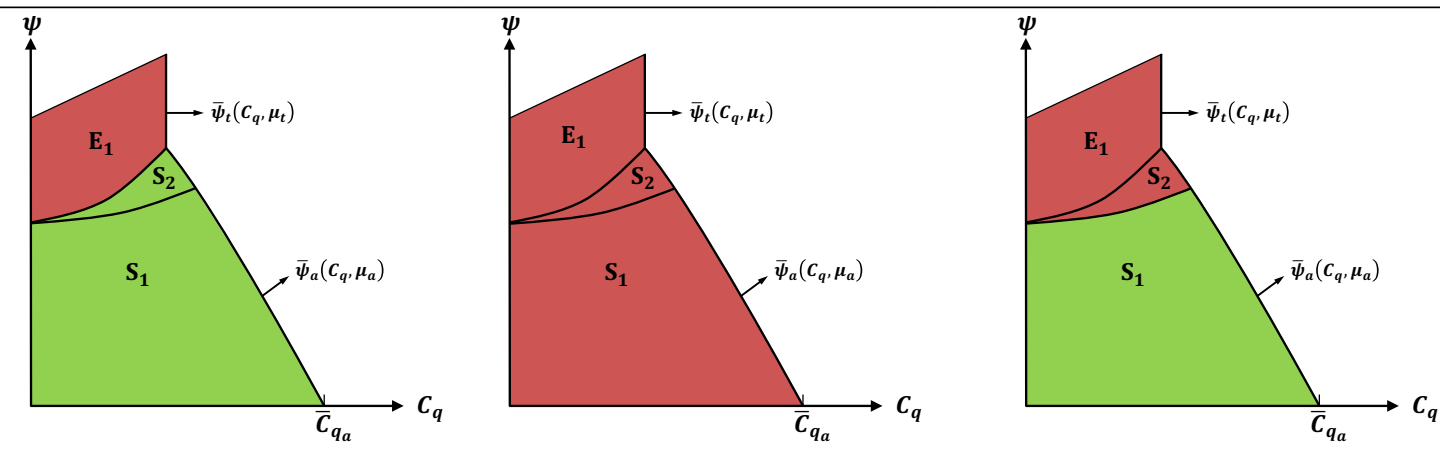

\begin{tabular}{cccc}
\hline Region & Manufacturer & $h$-type supplier & Channel \\
\hline $\mathrm{E}_{1}$ & $\pi_{\mathrm{QE}}>\pi_{\mathrm{QS}}$ & $\pi_{\mathrm{QE}}>\pi_{\mathrm{QS}}$ & $\pi_{\mathrm{QE}}>\pi_{\mathrm{QS}}$ \\
$\mathrm{S}_{1}$ & $\pi_{\mathrm{QE}}<\pi_{\mathrm{QS}}$ & $\pi_{\mathrm{QE}} \geq \pi_{\mathrm{QS}}$ & $\pi_{\mathrm{QE}}<\pi_{\mathrm{QS}}$ \\
$\mathrm{S}_{2}$ & $\pi_{\mathrm{QE}}<\pi_{\mathrm{QS}}$ & $\pi_{\mathrm{QE}}>\pi_{\mathrm{QS}}$ & $\pi_{\mathrm{QE}}>\pi_{\mathrm{QS}}$ \\
\hline
\end{tabular}

Notes: $C_{Q}=\left[p_{l}(1)-p_{l}(0)\right] r-c ; \bar{C}_{q_{a}}=\frac{\left[p_{\theta}(1)-p_{\theta}(0)\right] r-c}{2}$.

The different colored regions in the above figures correspond to whether or not the optimal QS contract is more beneficial than the optimal QE contract from each supply chain partner's perspective. Green areas denote that the optimal QS dominates the optimal QE; red regions denote that the optimal QE dominates the optimal QS; white regions denote an indifference between the optimal QS and QE contracts.

necessary quality requirements. However, an audit conducted under the optimal QS contract only ensures that the required effort is made, while the output itself may still fail. Therefore, the optimal QE contract lowers the direct cost in comparison with the optimal QS contract. This enables the manufacturer to conduct an inspection under the optimal QE contract for high values of $\psi$ compared to the optimal QS contract.

With respect to indirect channel loss: In general, the optimal QS contract leads to a lower indirect cost for the manufacturer than the optimal QE contract. This is due to the fact that the former 
is more powerful than the latter in incentivizing the supplier to make quality improvement efforts. There are two reasons behind this: First, incentives under the optimal QS contract are contingent on the effort made by the supplier, whereas those under the optimal QE contract are contingent on the actual output. This plays an important role especially when the cost of the quality improvement effort, denoted by $C_{q}$, is relatively high, because even though $C_{q}$ is paid as a contingent term under both optimal QE and QS contracts, the fact that its payment is tied to the effort rather than the output creates a relatively more powerful incentive for the supplier to make that effort under the optimal QS contract. Second, under the optimal QE contract, the production $\operatorname{cost} c$ is paid as a fixed term, whereas under the optimal QS contract, it is paid as a contingent term. This also makes the optimal QS contract more powerful than the optimal QE contract in incentivizing the supplier to make a greater quality improvement effort, especially when the production cost is relatively high, i.e., $c>\left[p_{\theta}(1)-p_{\theta}(0)\right] r$. Consequently, as shown in Table 5 , compared to the optimal QE contract, the optimal QS contract leads to a more reliable supply chain when the costs of production and quality improvement $-c$ and $C_{q}$ - are high (refer to the case when $C_{q}$ is high and $\left.c>\left[p_{\theta}(1)-p_{\theta}(0)\right] r\right)$.

With respect to information rent: Finally, the optimal QS contract is more beneficial to the manufacturer than the optimal QE contract due to its lower information rent. This is because the likelihood of a high-quality output from the supplier depends on his type, whereas the quality improvement effort made is the same for both $l$ - and $h$-type suppliers. Therefore, the optimal QE contract differentiates between the suppliers based on their extent of reliability, whereas the effortbased optimal QS contract does not. This in turn leads to information rent payment only under the optimal QE contract.

Comparison from the supplier's perspective: As opposed to the manufacturer's perspective, a comparison between the optimal QE and QS contracts from the supplier's perspective always leads to the former seeming more beneficial than the latter. There are two reasons for this: First, as mentioned above, the optimal QS contract gives the manufacturer more power on the supplier in comparison with the optimal QE contract. This enables the manufacturer to extract more surplus from the supplier under the optimal QS contract than under the optimal QE contract. Second, the optimal QE contract is type-contingent, whereas the optimal QS contract does not depend on the supplier's reliability. Being type-contingent, the optimal QE contract leads to the manufacturer paying information rent to the more reliable $h$-type in order to separate him from the less reliable l-type supplier. In contrast, under the optimal QS contract, the contingent payment is made to the supplier based on his effort rather than his reliability. This eliminates the need for paying the information rent; therefore, the more reliable $h$-type supplier is always worse off under the optimal QS contract. 
Nikoofal and Gümüş: Quality at the Source or at the End?

Comparison from the perspective of the supply chain as a whole: A comparison between the optimal QE and QS contract from the perspective of the supply chain as a whole is similar to its comparison from the perspective of the manufacturer. This is because when a contract type enables the manufacturer to implement the first-best outcome in a cost-efficient manner, it also leads to a more reliable supply chain at a minimum cost. There is a slight difference between the incentives of the manufacturer and of the entire supply chain in the cases of $S_{1}$ and $S_{2}$. This is due to the fact that the quality improvement effort is induced in pure strategies as per the optimal QE contract, while it is induced in mixed strategies as per the optimal QS contract. Since the former leads to a more reliable supply chain than the latter, the entire supply chain surplus is maximized under the optimal QE contract in regions $S_{1}$ and $S_{2}$ when the production cost $c$ is low and in region $S_{2}$ when $c$ is high.

\section{Model Extensions}

The analysis of the optimal QE and QS strategies so far is based on a number of assumptions. Three of them are as follows: i) either QE or QS contract is offered by the manufacturer, ii) the costs of audits and inspection tests are equal, and iii) in case of a defective product, the manufacturer does not incur any liability cost other than the loss of revenue. In this section, we relax them, one at a time, to study whether our individual and comparative analyses of the optimal QE and QS contracts are general enough and remain valid even under those circumstances.

\subsection{The Optimal Combined QE and QS Contracts}

In case of a joint inspection and audit, the manufacturer would offer a combined contract denoted by $\left(\omega_{\theta}, Y_{\theta}, E_{\theta}\right)$, where the contingent payment $Y_{\theta}$ is tied to the outcomes of both the audit and the inspection. More specifically, the manufacturer releases $Y_{\theta}$ subject to a combination of the following two conditions: (1) the manufacturer's audit confirms that the supplier's process improvement efforts $e_{\theta}$ match the efforts $E_{\theta}$ required by the contract, i.e., $e_{\theta}=E_{\theta}$; and (2) the inspection test confirms that the end product's quality is satisfactory. Let us suppose that the manufacturer wants to induce the $\theta$-type supplier to make quality improvement efforts, i.e., $E_{\theta}=1$. Depending on whether the manufacturer decides to audit the efforts and/or inspect the quality of the end product, she has four possible choices: (i) $A=1, T=1$; (ii) $A=1, T=0$; (iii) $A=0, T=1$; and (iv) $A=0, T=0$. The $\theta$ type supplier's decision is whether to make a process improvement effort, i.e., $e_{\theta}=0$ and $e_{\theta}=1$. The following table presents the strategic form of the joint inspection/audit sub-game, where the first and second expressions in each cell are the expected profits of the supplier and the manufacturer, respectively. 
Table 6 The supplier's (first expression) and manufacturer's (second expression) expected profits under the joint

\begin{tabular}{|c|c|c|c|c|}
\hline $\begin{array}{l}\text { Supplier's } \\
\text { efforts }\end{array}$ & $T=1 ; A=1$ & $T=0 ; A=1$ & $T=1 ; A=0$ & $T=0 ; A=0$ \\
\hline$e_{\theta}=1$ & $\begin{array}{c}\pi_{S}=\omega_{\theta}-c+ \\
{\left[p_{\theta}(1)+\mu_{t}\left(1-p_{\theta}(1)\right)\right] Y_{\theta}-C_{q}} \\
\pi_{M}=p_{\theta}(1) r-\omega_{\theta}-\psi_{t}-\psi_{a}- \\
{\left[p_{\theta}(1)+\mu_{t}\left(1-p_{\theta}(1)\right)\right] Y_{\theta}}\end{array}$ & $\begin{array}{c}\pi_{S}=\omega_{\theta}-c+Y_{\theta}-C_{q} \\
\pi_{M}=p_{\theta}(1) r-\omega_{\theta}-\psi_{a}-Y_{\theta}\end{array}$ & $\begin{array}{c}\pi_{S}=\omega_{\theta}-c-C_{q}+ \\
{\left[p_{\theta}(1)+\mu_{t}\left(1-p_{\theta}(1)\right)\right] Y_{\theta}} \\
\pi_{M}=p_{\theta}(1) r-\omega_{\theta}-\psi_{t}- \\
{\left[p_{\theta}(1)+\mu_{t}\left(1-p_{\theta}(1)\right)\right] Y_{\theta}}\end{array}$ & $\begin{aligned} \pi_{S} & =\omega_{\theta}+Y_{\theta}-c-C_{q} \\
\pi_{M} & =p_{\theta}(1) r-\omega_{\theta}-Y_{\theta}\end{aligned}$ \\
\hline$e_{\theta}=0$ & $\begin{array}{c}\pi_{S}=\omega_{\theta}-c+ \\
\mu_{a}\left[p_{\theta}(0)+\mu_{t}\left(1-p_{\theta}(0)\right)\right] Y_{\theta} \\
\pi_{M}=p_{\theta}(0) r-\omega_{\theta}-\psi_{t}-\psi_{a}- \\
\mu_{a}\left[p_{\theta}(0)+\mu_{t}\left(1-p_{\theta}(0)\right)\right] Y_{\theta}\end{array}$ & $\begin{array}{c}\pi_{S}=\omega_{\theta}-c+\mu_{a} Y_{\theta} \\
\pi_{M}=p_{\theta}(0) r-\omega_{\theta}-\psi_{a}- \\
\mu_{a} Y_{\theta}\end{array}$ & $\begin{array}{c}\pi_{S}=\omega_{\theta}-c+ \\
{\left[p_{\theta}(0)+\mu_{t}\left(1-p_{\theta}(0)\right)\right] Y_{\theta}} \\
\pi_{M}=p_{\theta}(0) r-\omega_{\theta}-\psi_{t}- \\
{\left[p_{\theta}(0)+\mu_{t}\left(1-p_{\theta}(0)\right)\right] Y_{\theta}}\end{array}$ & $\begin{array}{c}\pi_{S}=\omega_{\theta}+Y_{\theta}-c \\
\pi_{M}=p_{\theta}(0) r-\omega_{\theta}-Y_{\theta}\end{array}$ \\
\hline
\end{tabular}

We can employ the same three-step approach as in $\S 4$ and 5 to characterize the optimal combined contract under the joint audit and inspection game. Namely, first we analyze the Nash equilibria of the joint game, then characterize the optimal contract that would induce each equilibrium, and, finally, compare the optimal combined contracts and find the one that would maximize the expected profit of the manufacturer. To avoid repetition, here we focus on whether or not the combined contract would bring any marginal value over the optimal QE or QS contract, and, if so, under which conditions it can be strictly better than either of them. In order to simplify the analysis, below we assume that both $\psi_{t}=\psi_{a}=0$.

Proposition 5. Suppose $\psi_{t}=\psi_{a}=0$. Then, the quality improvement effort can be implemented only under the optimal combined QE and QS contract when both inspection and audit tests are not very accurate, i.e., $\mu_{t}>\bar{\mu}_{t}$ and $\mu_{a}>\bar{\mu}_{a}$. Furthermore, in this case, i.e., when $\mu_{t}>\bar{\mu}_{t}$ and $\mu_{a}>\bar{\mu}_{a}$, the region under which the optimal combined $Q E$ and $Q S$ contract strictly dominates over the optimal individual QE and QS contracts has been characterized in Figure 2.

As mentioned earlier, the Nash equilibrium in which the $\theta$-type supplier makes a quality improvement effort ceases to be an implementable strategy under both the QE and QS contracts when the degree of accuracy of both inspection and audit tests is low, i.e., $\mu_{t}>\bar{\mu}_{t}$ and $\mu_{a}>\bar{\mu}_{a}$, respectively. Surprisingly, the above Proposition shows that this is exactly when the combined strategy becomes valuable for the manufacturer, because by combining the information obtained from a joint inspection and audit, the manufacturer can boost the overall accuracy of the information. Thanks to the dual source of information, the manufacturer can induce the supplier to make quality improvement efforts even if the accuracy of each individual source is low.

That said, whether the quality improvement effort would be induced or not ultimately depends on the comparison between the agency costs incurred under the combined QE and QS contract. Similar to $\S \S 4$ and 5, our analysis shows that inducing a quality improvement effort helps the manufacturer 
Figure 2 Region in which the optimal combined contract strictly dominates over the individual optimal QE and QS contracts

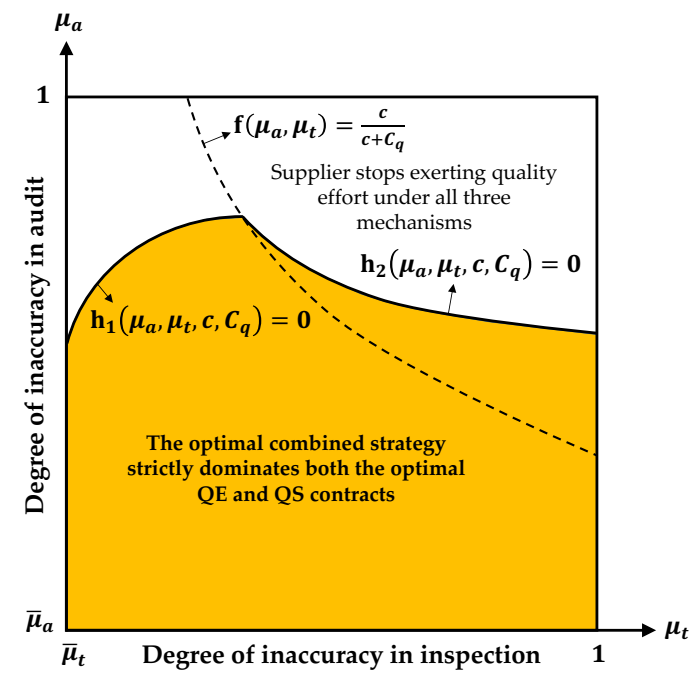

Notes. Refer to the Appendix for the derivation of the expressions for $\mathrm{f}\left(\mu_{a}, \mu_{t}\right), \bar{\mu}_{a}, \bar{\mu}_{t}, h_{1}\left(\mu_{a}, \mu_{t}, c, C_{q}\right)$, and $h_{2}\left(\mu_{a}, \mu_{t}, c, C_{q}\right)$.

eliminate the channel loss of indirect type as there is no cost associated with the inspection or audit; however, it leads to information rent. Delegating a detailed comparison to the Appendix, we show here that the higher the cost of quality improvement $C_{q}$, the lower the marginal benefit of the combined optimal contract for the manufacturer, compared with either the optimal QE or QS contract individually.

\subsection{Differing Costs of Inspections and Audits}

Even though in $\S 4$ and $\S 5$ we characterize the optimal QE and QS contracts under the general cost parameters $\psi_{t}$ and $\psi_{a}$, where $\psi_{t} \neq \psi_{a}$, we conduct a comparative analysis between the optimal QE and QS under the assumption of $\psi_{t}=\psi_{a}$ in $\S 6$. Eliminating this by considering either $\psi_{t}<\psi_{a}$ or $\psi_{t} \geq \psi_{a}$ does not lead to any further insight other than shifting the profit expressions and regions under the optimal QE and QS contracts. For example, increasing $\psi_{t}$ while keeping $\psi_{a}$ constant leads to two types of changes: First, ceteris paribus as $\psi_{t}$ increases, the manufacturer first begins to conduct inspections only occasionally and then stops conducting them altogether. As a result of this, the supplier does not make process improvement efforts under the optimal QE contract compared to under the optimal QS contract, which in turn leads to a channel loss. To summarize, the QS contract becomes more beneficial for a manufacturer as $\psi_{t}$ increases. This effect can be seen in Table 5 as the areas colored green (corresponding to the cases where QS is preferred over QE) become larger at the expense of areas colored red. 


\subsection{Manufacturer's Non-zero Liability Cost}

We assume that in case of a defective product, the manufacturer would incur only the loss of revenue $r$ per unit. In this section, we extend our base model by incorporating a non-zero liability cost in addition to $r$. Let $\mathscr{L}$ denote the manufacturer's total loss per unit that consists of $r$ and an additional liability cost. Note that our base model corresponds to the case where $\mathscr{L}=r$. As shown in the following proposition, we can extend both individual analyses of the optimal QE and QS contracts and their comparisons to include a non-zero liability cost as long as $\mathscr{L} \leq r+\psi_{t}$.

Proposition 6. The optimal QE and QS contracts with a non-zero liability cost are characterized in the Appendix as long as $\mathscr{L} \leq r+\psi_{t}$.

A further analysis of the optimal QE and QS contracts when $\mathscr{L} \leq r+\psi_{t}$ does not result in any new regions other than those characterized in Propositions 2 and 3 in $\S 4$ and $\S 5$, respectively. That said, both expressions in each region as well as the regions themselves are affected by $\mathscr{L}$, which in turn alters the comparison between the optimal QE and QS contracts. Given the limitations of space, we will focus here only on the main points.

As the total unit loss $\mathscr{L}$ increases, the region $Q E_{4}$ under the optimal QE contract (in which the manufacturer does not conduct an inspection) gets smaller, whereas the other regions (in which the manufacturer does conduct an inspection) get larger. This essentially implies that the manufacturer avoids incurring the potential loss that could be incurred by sending out defective products to customers. The manufacturer does so by offering the optimal QE contract and inspecting the end product rather than offering the optimal QS contract and auditing the supplier's efforts. Therefore, as $\mathscr{L}$ increases, the regions in which the manufacturer is better off using the optimal QE contract get bigger. We also briefly comment on the case in which $\mathscr{L}>r+\psi_{t}$. As opposed to $\mathscr{L} \leq r+\psi_{t}$, this case leads to new regions and hence complicates the comparison between the optimal QE and QS contracts. Even though understanding the impact of $\mathscr{L}$ on the comparison requires a more in-depth analysis, we expect that as $\mathscr{L}$ increases, the manufacturer would still be better off using the optimal QE contract.

\section{Conclusions}

Despite the many benefits of outsourcing, the increase in information asymmetry between the parties involved in a supply chain and quality-related issues resulting from a lack of control have become a primary concern for organizations. In this paper, we explored the value of contracting for a firm that has to contract work to a supplier whose true state of delivery quality is not observable. We analyzed two contractual mechanisms through which a manufacturer can interact with such suppliers. In the first mechanism, the manufacturer offers the supplier a menu of contracts that depend on the 
Nikoofal and Gümüş: Quality at the Source or at the End?

product's end product (the Quality-at-the End (QE) contract). In the second, the manufacturer gives incentives based on the supplier's quality improvement efforts at source (the Quality-at-theSource (QS) contract). The characterization and comparison of optimal contracts under these two mechanisms yielded insights regarding the impact of tying incentives and quality inspections to source- versus end-quality on the reliability of the supply chain, decisions regarding equilibria, and payoffs to individual supply chain parties, as well as the supply chain as a whole. Building on this comparative analysis, we summarized the conditions under which each type of contract would be the most likely equilibrium strategy for a manufacturer in Table 7.

Table 7 Conditions for the most likely equilibrium contracting strategy for a manufacturer

\begin{tabular}{l|l}
\hline \hline Output-based QE Contract & Action-based QS Contract \\
\hline \hline - Low production cost & $\bullet$ High production cost \\
- Low cost of quality improvement & $\bullet$ High cost of quality improvement \\
- High cost of inspection/audit & $\bullet$ Low cost of inspection/audit \\
- Low information asymmetry & $\bullet$ High information asymmetry \\
- High accuracy in inspection & $\bullet$ High accuracy in audit \\
\hline \hline
\end{tabular}

Our analyses established that when the supplier incurs a higher proportion of the cost related to quality improvement or production, it is optimal for the manufacturer to use the mechanism involving action-based incentives and inspections. This is because under the optimal QS contract, the supplier is completely subsidized, as long as he makes quality improvement efforts, irrespective of whether the final outcome satisfies the quality specifications. This greatly incentivizes the supplier to make quality improvement efforts. We also demonstrated that when the manufacturer incurs a higher (resp. less) cost related to inspection (resp. information asymmetry), it would be optimal for this manufacturer to employ the mechanism involving output-based incentives and inspections. Compared to the optimal QS, the optimal QE contract helps to shift the risk from the manufacturer to the supplier. This lowers the implementation cost from the manufacturer's perspective, because she releases the contingent payment only after the final outcome has been shown to conform to the quality specifications. Finally, we analyzed the marginal value of using a combined contract as opposed to the optimal QE or QS contracts alone. Our analysis showed that a combined contract strictly dominates over the optimal QE and QS contracts when the accuracy of inspections and audits is relatively low. This is because the manufacturer can combine the QE and QS contracts to increase the accuracy of the inspection and audit tests, which in turn enables her to induce the supplier to make quality improvement efforts. 
The model presented in this paper can be extended in multiple directions. In this paper, we focused only on uncertainty with regard to quality. However, we can also show that both the model and its analyses can be applied to a similar situation in which the supplier's actions lead to uncertainty about the product's quantity. Another application is to consider the impact of multiple (possibly continuous) reliability types and efforts for the supplier. Even though such an analysis would be considerably more complicated, we expect that the qualitative nature of our results under two-type and two-effort cases would hold in these situations. Also, we assume that the cost of quality improvement efforts is type-independent for a supplier. Setting aside this assumption would lead to two types of information asymmetries resulting from differences between suppliers in terms of their extent of reliability and cost. We intuit that this would lead to a higher (resp. less) agency cost under the optimal QE contract than under the optimal QS contract. Thus, the optimal QE contract becomes less (resp. more) beneficial than the optimal QS contract from the manufacturer's perspective, when a more reliable supplier is also more (resp., less) cost efficient in quality improvement.

Last but not least, we believe that managing quality under information asymmetry has become an increasingly important issue for many companies as they expand their supply bases locally and globally. We hope that our model will contribute to understanding the key factors of this issue.

\section{References}

Erin Anderson and Richard L Oliver. Perspectives on behavior-based versus outcome-based salesforce control systems. The Journal of Marketing, pages 76-88, 1987.

S. Anthon, S. Garcia, and A. Stenger. Incentive contracts for natura 2000 implementation in forest areas. Environmental and Resource Economics, 46(3):281-302, 2010.

V. Babich and C. S. Tang. Managing opportunistic supplier product adulteration: Deferred payments, inspection, and combined mechanisms. Manufacturing \& Service Operations Management, 14(2):301$314,2012$.

S. Baiman, P.E. Fischer, and M.V. Rajan. Information, contracting, and quality costs. Management Science, pages 776-789, 2000.

S. Baiman, S. Netessine, and H. Kunreuther. Procurement in supply chains when the end-product exhibits the weakest link property. http:/grace. wharton. upenn. edu/risk/downloads/Baima, (2ONetessin), 2003.

S. Baiman, S. Netessine, and H. Kunreuther. Procurement in supply chains when the end-product exhibits the weakest link property. University of Pennsylvania. Working Paper, February, 2004.

K. R. Balachandran and S. Radhakrishnan. Quality implications of warranties in a supply chain. Management Science, 51(8):1266-1277, 2005.

Amiya K Basu, Rajiv Lal, Venkataraman Srinivasan, and Richard Staelin. Salesforce compensation plans: An agency theoretic perspective. Marketing science, 4(4):267-291, 1985. 
G. P. Cachon. Supply chain coordination with contracts. Handbooks in operations research and management science, 11:229-340, 2003.

G. P. Cachon and F. Zhang. Procuring fast delivery: Sole sourcing with information asymmetry. Management Science, 52(6):881, 2006.

G. H. Chao, S. M. R. Iravani, and R. C. Savaskan. Quality improvement incentives and product recall cost sharing contracts. Management Science, 55(7):1122-1138, 2009.

A. Chaturvedi and V. Martinez-de Albeniz. Optimal procurement design in the presence of supply risk. Manufacturing \& Service Operations Management, 13(2):227-243, 2011.

C. J. Corbett, D. Zhou, and C. S. Tang. Designing supply contracts: Contract type and information asymmetry. Management Science, 50(4):550-559, 2004.

Anne T Coughlan and Subrata K Sen. Salesforce compensation: Theory and managerial implications. Marketing Science, 8(4):324-342, 1989.

J. H. Dyer, D. S. Cho, and W. Chu. Strategic supplier segmentation: The next "best practice" in supply chain management. California Management Review, 40:57-77, 1998.

M. Gümüs, S. Ray, and H. Gurnani. Supply side story: Risks, guarantees, competition and information asymmetry. Management Science, 58 (9):1694-1714, 2012.

M. Harley. Volvo recalls over 140k 2001-2005 and 2010 my vehicles over fuel system issues. Autoblog, http://www.autoblog.com/volvo/recalls/, Decebmer 2009.

Kissan Joseph and Alex Thevaranjan. Monitoring and incentives in sales organizations: An agency-theoretic perspective. Marketing Science, 17(2):107-123, 1998.

M. Kaya and O. Özer. Quality risk in outsourcing: Noncontractible product quality and private quality cost information. Naval Research Logistics, 56(7):669-685, 2009.

S. H. Kim and S. Netessine. Collaborative cost reduction and component procurement under information asymmetry. Management Science, 59(1):189-206, 2013.

J. Knowdell. The benefits and disadvantages of contract manufacturing. 2010.

J. J. Laffont and D. Martimort. The theory of incentives: the principal-agent model. Princeton Univ Pr, 2002.

J. J. Laffont and J. Tirole. Using cost observation to regulate firms. The Journal of Political Economy, pages 614-641, 1986.

Marianna Makri, Peter J Lane, and Luis R Gomez-Mejia. Ceo incentives, innovation, and performance in technology-intensive firms: a reconciliation of outcome and behavior-based incentive schemes. Strategic Management Journal, 27(11):1057-1080, 2006.

Kevin J Murphy. Executive compensation. Handbook of labor economics, 3:2485-2563, 1999. 
R. B. Myerson. Optimal coordination mechanisms in generalized principal-agent problems. Journal of mathematical economics, 10(1):67-81, 1982.

O. Özer and G. Raz. Supply chain sourcing under asymmetric information. Production and Operations Management, 20(1):92-115, 2011.

Jagmohan S Raju and V Srinivasan. Quota-based compensation plans for multiterritory heterogeneous salesforces. Management Science, 42(10):1454-1462, 1996.

D. J. Reyniers and C. S. Tapiero. The delivery and control of quality in supplier-producer contracts. Management Science, 41:1581-1589, 1995.

Huaxia Rui and Guoming Lai. Sourcing with deferred payment and inspection under supplier product adulteration risk. Production and Operations Management, 24(6):934-946, 2015.

CFO Research Services. Physical risks to the supply chain: the view from finance. Report, 2009.

Y. Sheffi. The resilient enterprise: overcoming vulnerability for competitive advantage. MIT Press Books, 2005.

Dean H Stamatis. Failure mode and effect analysis: FMEA from theory to execution. Asq Press, 2003.

C. S. Tang. Making products safe: process and challenges. International Commerce Review, 8(1):48-55, 2008.

B. Tomlin. Impact of supply learning when suppliers are unreliable. Manufacturing \& Service Operations Management, 11(2):192-209, 2009.

Volvo. Supplier quality assurance manual. Technical report, 2010.

Z. B. Yang, G. Aydin, V. Babich, and D. R. Beil. Supply disruptions, asymmetric information, and a backup production option. Management Science, 55(2):192-209, 2009.

Z. B. Yang, G. Aydin, V. Babich, and D. R. Beil. Using a dual-sourcing option in the presence of asymmetric information about supplier reliability: Competition vs. diversification. Manufacturing \& Service Operations Management, 14(2):202-217, 2012. 